Estudios sobre armas antiguas, arte militar $\mathrm{y}$ vida cultural en oriente y occidente XXIX (2009), pp. 93-120 ISSN: 0436-029X

\title{
LE SIEGE D'AZAILA (TERUEL): UNE RELECTURE CRITIQUE DES INDICES ARCHEOLOGIQUES
}

\author{
THE SIEGE OF AZAILA (TERUEL): A CRITICAL RE-READING \\ OF THE ARCHAEOLOGICAL EVIDENCE
}

POR

David Hourcade ${ }^{*}$

\section{Resume - Abstract}

On considère généralement que, lors des guerres sertoriennes, le site de Cabezo de Alcalá (Azaila, Teruel) a subi un siège qui aurait conduit à son abandon. Or, malgré l'apparente accumulation de preuves, une relecture critique des vestiges découverts permet de douter de la réalité de ce conflit. Ainsi, aucune défense urbaine ne semble avoir été ajoutée par la communauté et les «barricades» ne sont peut-être que des pierriers d'époque moderne. De même, les deux catapultes de torsion et les armes de poing — censées prouver l'âpreté du combat et la résistance des habitants - ne sont vraisemblablement que des anciens trophées, alors que les boulets découverts pourraient provenir de l'arsenal municipal. Enfin, il est probable que le talus situé au sud de la ville haute ne soit pas une rampe d'assaut construite par les assaillants, mais une simple rampe d'accès aménagée postérieurement pour rendre l'accès au sommet du site plus aisé.

It is generally considered that during the Sertorian Wars, the site of Cabezo de Alcalá (Azaila, Teruel) underwent a siege that would have led to its abandonment. But, despite the apparent accumulation of evidence, a critical re-reading of the traces discovered leaves the reality of this conflict open to doubt. No urban defence seems to have been built by the community and the «barricades» are perhaps no more than mounds of stones of recent date. Moreover, the two torsion catapults and hand-held weapons - considered evidence of heavy fighting and the inhabitants' resistance - are probably only ancient trophies, while the artillery projectiles discovered could come from the municipal arsenal. Finally, the slope situated to the south of the upper city is probably not an assault ramp, but a simple embankment, later converted to provide easier access to the summit of Cabezo.

\section{Mots-Cles - Keywords}

Azaila; Hispanie tardo-républicaine; guerres sertoriennes; siège; armes; catapultes; trophées; agger.

Azaila; Late Republican Spain; Sertorian wars; siege; weapons; catapults, trophies; agger.

* Doctorant Institut Ausonius, Université Michel de Montaigne, Bordeaux 3, France. e-mail: davidhourcade@ yahoo.fr. 
L'agglomération antique d'Azaila ${ }^{1}$ (Cabezo de Alcalá, Azaila, Teruel) est — malgré sa petite taille et le fait que son nom antique demeure encore incertain ${ }^{2}$ - l'un des sites d'époque tardo-républicaine les plus célèbres de la péninsule Ibérique ou, du moins, de son quart nordest. Sa renommée est due, avant tout, aux grands travaux de dégagement dont elle a fait l'objet durant toute la première moitié du XX $\mathrm{XX}^{\mathrm{e}}$ siècle. Dès 1919, J. Cabré y a, en effet, mis au jour les restes d'une agglomération ibère extrêmement bien conservée (fig. 1) ${ }^{3}$. Par la suite, le site a fait l'objet de quelques études ponctuelles —essentiellement dans les années $1960^{4}$ — avant que les travaux de M. Beltrán Lloris n'assurent définitivement sa renommée dès la fin des années 1970 et lors des deux décennies suivantes ${ }^{5}$. Depuis, le site, qui a fait l'objet de divers travaux de mise en valeur et de consolidation ${ }^{6}$, tient une place particulière dans l'étude de la romanisation de la vallée de l'Èbre à la fin de la République'. Ainsi, l'organisation, la nature et la datation généralement proposée des vestiges mis au jour ${ }^{8}$ en font, pour la plupart des chercheurs, un site-témoin essentiel dans la compréhension du phénomène d'adoption précoce de schémas urbains méditerranéens dans la région ${ }^{9}$. En effet, les structures dégagées, qui dateraient principalement des $\mathrm{II}^{\mathrm{e}}$ et $\mathrm{I}^{\mathrm{er}}$ siècle a.C. ${ }^{10}$, présentent à la fois des marques très nettes

1 Cet article reprend les conclusions d'une communication présentée le 24 novembre 2007 à la Casa de Velázquez (Madrid), lors de la Table Ronde intitulée «La guerre et ses traces dans la péninsule Ibérique à l'époque de la conquête romaine: approches méthodologiques». Une réflexion plus méthodologique et générale est en cours de publication dans un numéro spécial de la revue Salduie (Hourcade, à paraître). Merci à Fernando Quesada de m'avoir invité à publier, ici, ces quelques remarques. Merci également à Pierre Aupert, François Cadiou, Olivier Dupont, Laurent Lamoine, Stéphane Lebreton et Pierre Moret pour leurs suggestions et commentaires.

2 Jusqu'au début de la seconde moitié du $\mathrm{XX}^{\mathrm{e}}$ siècle, on considérait qu'Azaila correspondait au site primitif de l'agglomération de Kelse/Celsa. Il aurait été abandonné dans les années 40 a.C. —c'est-à-dire au moment de la fondation de la colonia Victrix Iulia Lepida/Celsa - , au profit de celui de «Las Eras» (Velilla del Ebro, Saragosse), situé quelques kilomètres plus loin. Par la suite, cependant, les études menées sur ces deux sites, ainsi que la relecture des données chronologiques d'Azaila (Beltrán Lloris, 1984b), avaient conduit la plupart des chercheurs à vieillir la date d'abandon du premier site et à abandonner, par conséquent, cette hypothèse. Pourtant, l'attribution traditionnelle du site vient récemment de retrouver une certaine légitimité. En effet, A. Gorgues (2005: 430-433) propose de façon convaincante, à la suite du réexamen du mobilier mis au jour lors des fouilles de Cabré, de rabaisser la date d'abandon du site. Il en déduit également que rien ne s'oppose donc plus à ce qu'Azaila corresponde véritablement au site préromain de Celsa.

3 Cabré, 1925; Cabré, 1929; Cabré, 1941 et Cabré, 1944.

4 Beltrán Lloris, 1964; Beltrán Martínez, 1966.

5 Beltrán Lloris, 1976; Beltrán Lloris, 1984a; Beltrán Lloris, 1984b; Beltrán Lloris et al., 1995.

6 Franco et Hernández, 2004-2005.

7 Beltrán Martínez, 1987; Beltrán Lloris, 1990; Asensio Esteban, 1995: 146-167; Beltrán Lloris et al., 2000; Sillières, 2001; Barrandon, 2005.

8 On pense notamment au pavage des rues, aux thermes qui s'élèvent à proximité de la poterne orientale de l'acropole, au temple dit «in antis» qui se dresse sur le sommet de la colline, ainsi qu'au groupe de sculptures en bronze que Cabré a découvert à l'intérieur (Nony, 1969).

9 Je ne discuterai pas ici du bien-fondé de cette théorie. Elle me semble cependant désormais à envisager avec précaution. Déjà, dès le milieu des années 1980, certains chercheurs s'étonnaient de la place importante qui était faite à ce site. Ainsi, F. Burillo considérait qu'il était, un peu à tort, «hipervalorado» (Burillo, 1986: 5). De plus, on doit reconnaître que, faute de fouilles stratigraphiques fines, les datations avancées restent relativement subjectives. Ainsi, en ce qui concerne la datation des thermes, par exemple, il faut désormais tenir compte des critiques récemment formulées par A. Ribera et C. Marín. Selon eux, il conviendrait d'abaisser la date de construction de l'édifice au milieu du Ir $^{\text {r }}$ siècle a.C. (Ribera et Marín, 2004-2005: 284-285). Son plan, ainsi que les structures mises au jour à l'intérieur leur font douter de sa datation précoce de la fin du II ${ }^{\mathrm{e}}$ siècle a.C. Si, comme cela me semble le plus probable, la nouvelle datation devait être acceptée, le site perdrait alors une grande part de sa spécificité. En fait, comme on le verra plus loin, tout dépend essentiellement de la date d'abandon de l'agglomération.

10 La chronologie du site reste floue, surtout en ce qui concerne les débuts de son occupation. Cependant, l'ensemble des chercheurs s'accorde à penser que la majeure partie de son urbanisme, tel qu'il a été fossilisé, date, au plus tard, de la fin du II ${ }^{\mathrm{e}}$ siecle ou du début du I ${ }^{\text {er }}$ siecle a.C. (Beltrán Lloris, 1964; Beltrán Lloris, 1984b: 144-145; Moret, 1996: 422; Gorgues, 2005: 430-433). Cela ne signifie pas que le tissu urbain soit resté inchangé durant le demi-siècle suivant. Les thermes ont, par exemple, pu être ajoutés par la suite. 


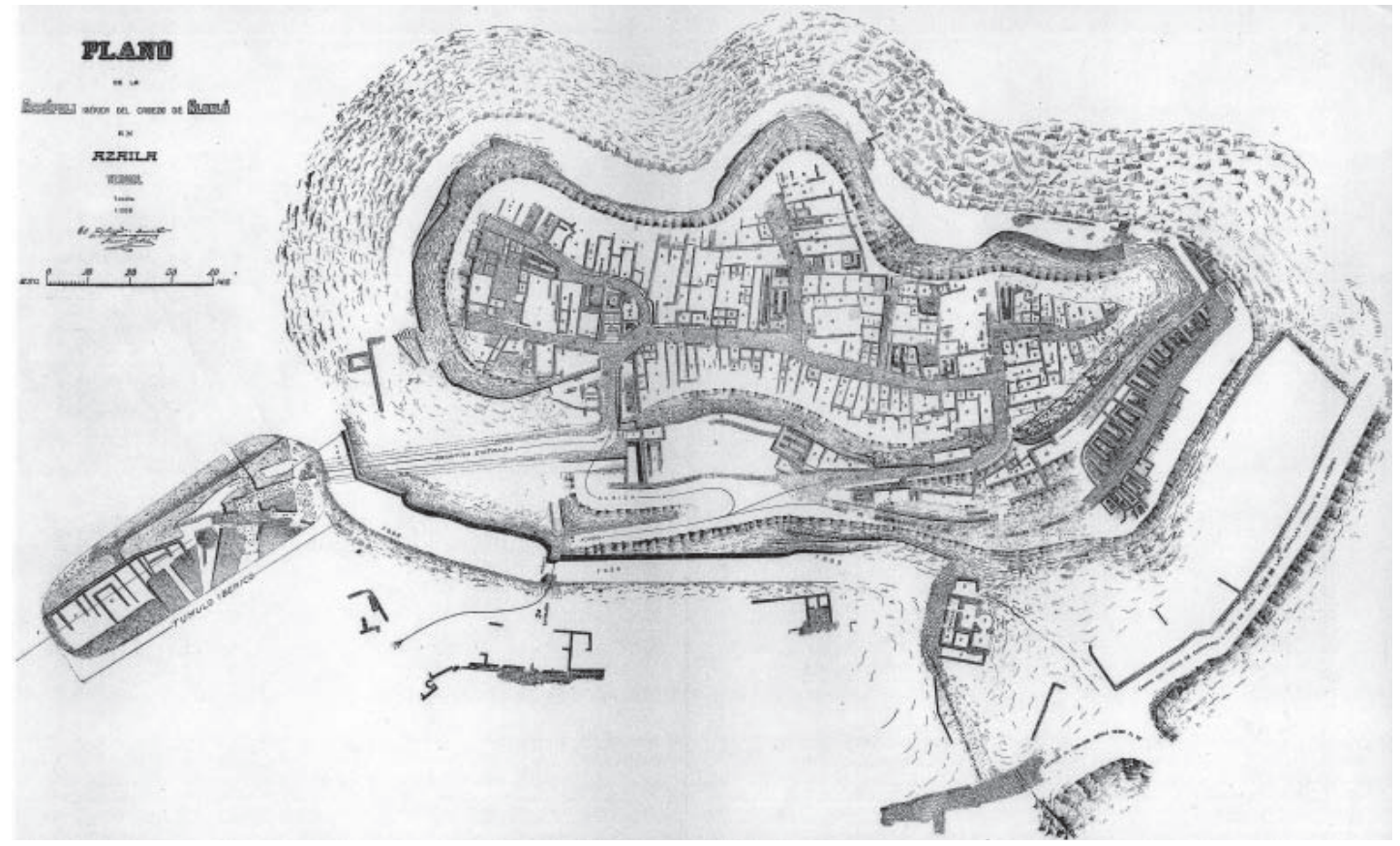

Figure 1. Plan du site d'Azaila (Cabezo de Alcalá, Azaila, Teruel) (d'après Cabré, 1944: fig. 1).

d'emprunts au monde italique et d'autres, plus traditionnelles, au monde indigène. Ainsi, bien que l'agglomération pérégrine couvre une superficie totale inhabituellement grande de plus de 10 ha et qu'elle montre des traces de «modernité» dans son urbanisme, elle reprend pourtant un schéma plus classique pour la région et pour l'époque ${ }^{11}$. Elle s'articule en effet autour de deux pôles distincts. La «ville haute», ou acropole, s'élève sur le sommet du «Cabezo de Alcalá», dans la partie occidentale du site, alors que la «ville basse», ouverte, s'étend en contrebas, au sud et à l'est (fig. 1 et 2). L'acropole, qui ne couvre qu'une faible superficie d'environ 1,5 à 2 ha, est la seule partie fortifiée de l'agglomération.

La seconde raison qui concourt à la notoriété de ce site tient à l'apparente accumulation de vestiges militaires qu'on y a découverts. Ils ont conduit les chercheurs à formuler l'hypothèse d'un siège — non attesté par les sources littéraires — qui aurait été fatal à l'agglomération ${ }^{12}$. Ainsi, depuis les années 1980, on considère que l'acropole d'Azaila aurait été prise et détruite au cours des guerres sertoriennes ${ }^{13}$. Pour étayer une telle hypothèse, les archéologues s'appuient sur une série de découvertes qu'ils interprètent d'un point de vue militaire. Selon eux ${ }^{14}$, les habitants auraient tout d'abord cherché, en vue du siège, à renforcer leurs défenses en ajoutant des lignes de «pierres plantées» en avant du fossé sud-est (fig. 3) ${ }^{15}$. Malheureusement, ces précautions n'auraient pas suffi et, une fois la muraille de la ville franchie par les

11 Asensio Esteban, 1995.

12 Sur les caractéristiques et les techniques des guerres de siège, on se reportera à Adcock, 1940; Sauvage, 1991; Campbell, 2003a; Campbell, 2005; Saéz Abad, 2003 et 2005.

13 Beltrán Lloris, 1984b; Beltrán Lloris, 1990; Beltrán Lloris et al., 1995; Romeo, 2004; Sáez Abad, 2005; Saéz Abad 2006.

14 On trouvera une proposition de restitution détaillée du déroulement du siège et de la prise de la ville dans Romeo, 2004.

15 Beltrán Lloris et al., 1995: 175; Romeo, 2004. 


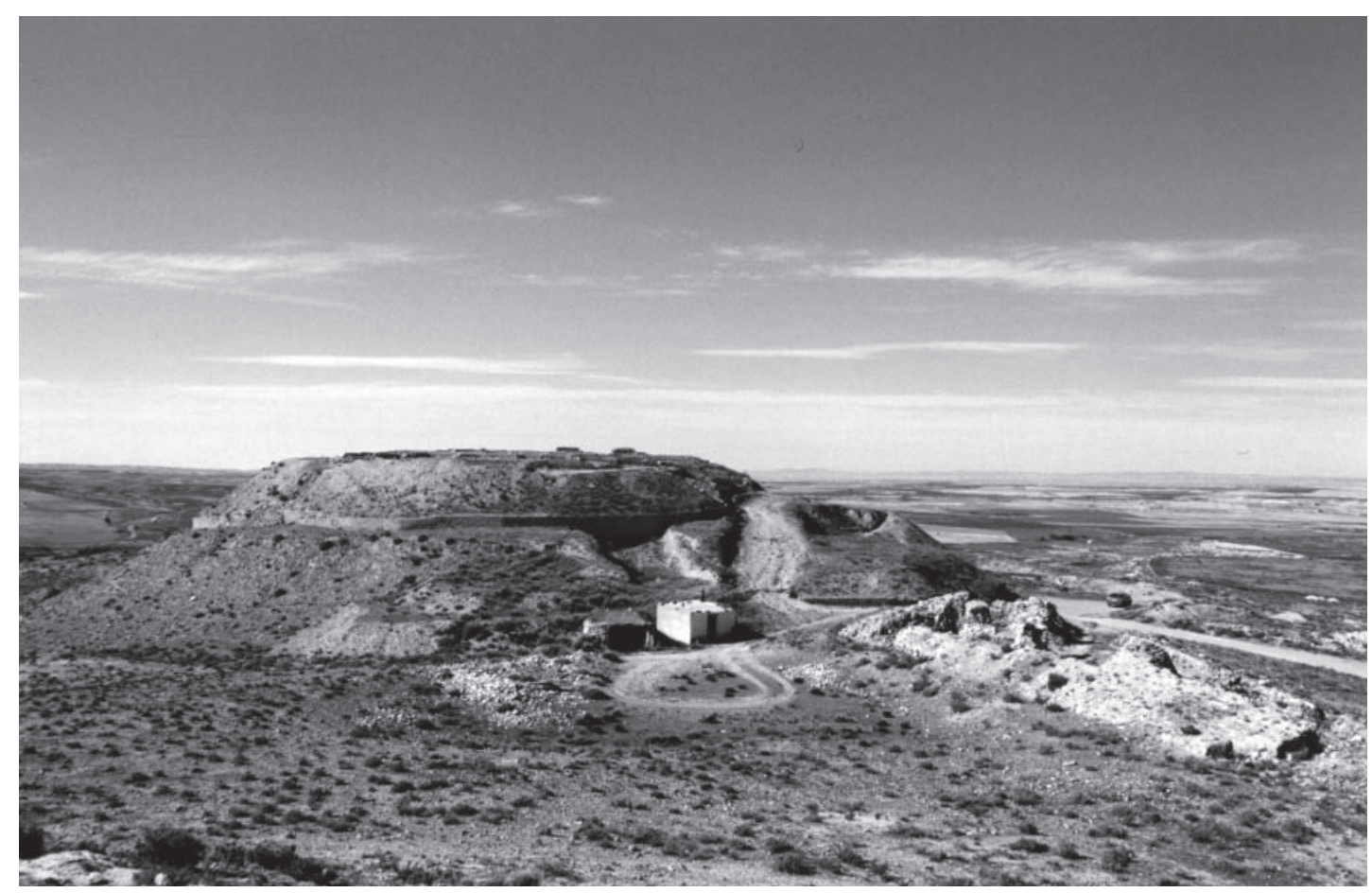

Figure 2. Vue de l'acropole d'Azaila depuis le sud-ouest (cl. D. Hourcade).

troupes ennemies, les assiégés se seraient réfugiés au sommet de l'acropole pour y livrer un dernier combat acharné (fig. 4). Les armes et les projectiles mis au jour sur le site témoigneraient de son âpreté ${ }^{16}$. De plus, afin de ralentir l'avancée des troupes romaines, les habitants de l'agglomération auraient tenté d'élever une série de barricades en travers des rues de la ville haute ${ }^{17}$. Ils auraient aussi transformé quelques lieux, dont le temple dit «in antis» et une maison aristocratique, en derniers bastions de résistance armée. C'est du moins ce que la découverte de deux pièces d'artillerie légère dans ces édifices démontrerait aux yeux de la plupart des chercheurs ${ }^{18}$. Mais ce sont surtout les prétendus travaux de terrassement réalisés par les assaillants qui font l'originalité du site. En effet, au sud-est du Cabezo, les fouilles de J. Cabré ont permis la mise au jour d'un grand talus d'environ $75 \mathrm{~m}$ de long et 26 à $27 \mathrm{~m}$ de large. D’abord identifiée comme un «tumulus ibérique», cette structure a été interprétée, depuis, comme une rampe d'assaut (fig. 1 à 3, 9 à 11) ${ }^{19}$. Ce serait grâce à elle que les troupes romaines auraient pu franchir la muraille et s'emparer de la ville haute ${ }^{20}$. C'est d'ailleurs à cause de ce supposé agger que le site d'Azaila est parfois surnommé «la Masada de Hispania» ${ }^{21}$.

16 Il s'agit de plusieurs armes de poing (épées, poignards) et de nombreuses armes de jets et de projectiles (lances, flèches, balles de fronde en plomb, boulets de pierres). On en trouvera la liste dans Beltrán Lloris, 1976 ou Sáez Abad, 2006.

17 J. Cabré pensait en avoir retrouvé deux témoignages en travers des rues A et C (Cabré, 1929).

18 Il s'agit de deux catapultes de torsion de type scorpio. Pour leur description détaillée, voir García Díez, 2002. On verra plus loin que l'interprétation qu'il fait de ces armes est bien différente de celle des autres chercheurs.

19 Beltrán Lloris, 1976; Beltrán Lloris et al., 1995.

20 Romeo, 2004; Sáez Abad, 2005: 161.

21 Ainsi, c'est par cette formule, et avec une pointe d'humour, que J. Gómez-Pantoja m'a invité à présenter ma communication lors de Table Ronde «La guerre et ses traces» tenue à la Casa de Velázquez de Madrid. 


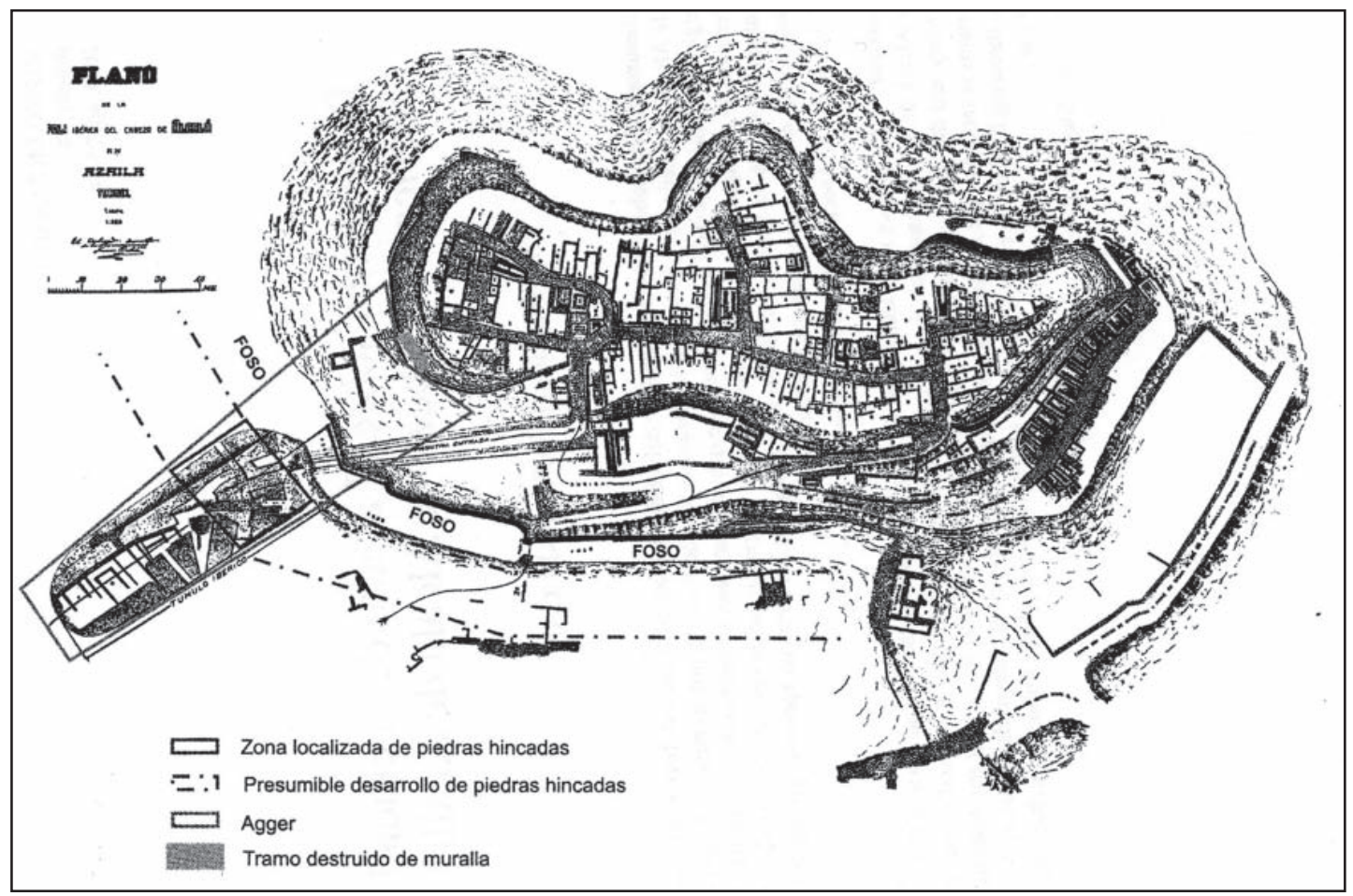

Figure 3. Plan des fortifications mises en place au cours du siège supposé de l'acropole d'Azaila (d'après Romeo, 2004: fig. 1).

Comme on le voit, la fin de l'agglomération semble bien connue. La découverte de ces nombreux vestiges militaires, ou supposés tels, paraît confirmer l'interprétation proposée par la majorité des chercheurs et ne laisser que peu de place aux doutes. Pourtant, malgré cette apparente accumulation d'indices — ajout de fortifications ponctuelles, présence d'armes et traces de grands travaux de terrassement- je pense qu'une relecture critique de l'ensemble des vestiges mis au jour permet d'aboutir à une conclusion différente ${ }^{22}$. Selon moi, le siège de la ville n'a pas eu lieu, ou, du moins, pas à l'époque ni dans les circonstances généralement évoquées. Il n'a d'ailleurs pas été fatal à l'agglomération. De plus, il me semble possible de proposer une interprétation civile, civique $^{23}$ et pacifique, ou du moins démilitarisée, de l'ensemble des «témoins» du supposé siège de la ville.

22 Pour mémoire, rappelons également que les archéologues médiévistes prendront en compte que, pour qu'une destruction d'habitats soit considérée comme d'origine militaire, il faut avoir mis au jour quatre types particuliers de vestiges: des traces généralisées d'incendie; beaucoup de mobilier quotidien abandonné sur place; de nombreuses armes, notamment des projectiles; et des squelettes d'animaux domestiques violemment tués (Pesez et Piponnier, 1988). On se rend compte qu'Azaila ne remplit qu'un seul de ces quatre critères. Seules les armes y ont été mises au jour.

${ }^{23}$ La défense de la communauté fait partie des premiers devoirs du citoyen. La fortification de la ville, ainsi que l'entretien d'un arsenal municipal, peuvent donc, en ce sens, être compris comme un acte civique. Ils n'impliquent cependant pas un état de guerre déclarée. 
Ainsi, la réalité du siège et de la prise d'Azaila ne me semble reposer, avant tout, que sur une série de présupposés historiographiques. C'est, selon moi, la conclusion à laquelle permettent d'aboutir le réexamen des données chronologiques, la relecture des fortifications du site, ainsi que la réinterprétation du contexte de découverte des armes mises au jour.

Le premier problème auquel on se trouve confronté, au moment d'étudier les défenses mises en place par les habitants d'Azaila, est celui de leur datation. Cette question est en fait intimement liée à celle de la date d'abandon du site et de son hypothétique siège.

S'il ne fait aucun doute que les principales fortifications de la ville préexistaient à ce dernier, on doit néanmoins remarquer que leur datation est mal assurée. En effet, faute de fouilles stratigraphiques minutieuses de l'enceinte ${ }^{24}$, la datation des remparts ne peut être déduite que de ce que l'on sait de l'histoire du site. Ainsi, dans un premier temps, on avait découpé l'histoire du site en trois phases principales ${ }^{25}$. La «Ciudad I» correspondrait à une phase préromaine; la «Ciudad II» serait la ville du II $^{\mathrm{e}}$ siecle a.C. et la «Ciudad III» celle du ${ }^{\text {er }}$ siecle a.C. Ce découpage chronologique — qui reflétait en fait en partie les grands conflits que la vallée de l'Èbre avait connus à la fin de la République — avait d'abord été admis par M. Beltrán Lloris ${ }^{26}$. Mais, après réexamen du mobilier céramique provenant des dernières couches d'occupation du site, il était ensuite arrivé à la conclusion qu'il n'existait pas de troisième phase. Selon lui, Azaila aurait été abandonnée entre 76 et 72 a.C. à la suite du siège de la ville par les troupes de Pompée, lors de la répression de l'épisode sertorien ${ }^{27}$. Au final, si l'on s'en tient à cette chronologie du site, les murailles de la ville auraient donc été construites lors de la phase de la «Ciudad II», c'est-à-dire dans le courant du $\mathrm{II}^{\mathrm{e}}$ siecle a.C. ${ }^{28}$ Les défenses ajoutées lors du dernier siège du site dateraient, quant à elles, des années 70 a.C.

Pourtant, deux études céramologiques, menées indépendamment ${ }^{29}$, viennent récemment et de façon convaincante de mettre en doute la date traditionnellement proposée pour l'abandon du site. Ainsi, les travaux que A. Ribera et C. Marín, d'une part, et A. Gorgues, de l'autre, ont menés sur le mobilier provenant des derniers niveaux d'occupation du site démontrent que la ville a, en fait, été occupée au moins jusqu'aux années 50-40 a.C. Azaila n'a donc pas pu être abandonnée à la suite d'un siège survenu à la fin des années 70 a.C. Cette nouvelle datation, qui reprend celle proposée jusqu'aux années $1970^{30}$, permet d'ailleurs de mieux s'accorder avec les dates avancées par d'autres chercheurs au sujet du groupe de sculptures en bronze découvert par J. Cabré dans le temple dit «in antis» ${ }^{31}$. En effet, depuis les travaux de Beltrán Lloris ${ }^{32}$, on considère qu'il

24 En ce sens, il est regrettable que les récents sondages réalisés sur le rempart oriental, lors des travaux de consolidation de 2002, n'aient pas permis de le dater (Franco et Hernández 2004-2005). À ma connaissance, le matériel recueilli, qui pourrait appartenir à un dépôt de fondation, n'a pas encore été étudié en détail. Il constituerait pourtant un marqueur chronologique important.

25 D'après Beltrán (1964), la «Ciudad I» daterait de l'époque comprise entre 550 et 197/195 a.C. Elle aurait été détruite par les troupes de Caton. La «Ciudad II» aurait quant à elle été détruite par les armées de Pompée, lors des guerres sertoriennes. Enfin, la «Ciudad III» aurait été assiégée et détruite par César vers 49 a.C.

26 Beltrán Lloris 1976; Beltrán Lloris 1984b.

27 Notons pourtant que les sondages réalisés dans les années 1970 n'avaient révélé aucune trace de destruction violente ni d'incendie généralisé.

28 Ces conclusions restent cependant hypothétiques. Par prudence et en fonction des caractéristiques techniques des structures, il me semble préférable de penser qu'elles datent d'une période plus imprécise comprise entre le $\mathrm{III}^{\mathrm{e}}$ et le $\mathrm{II}^{\mathrm{e}}$ siècle a.C.

29 Ribera et Marín, 2004-2005; Gorgues, 2005: 430-433.

30 Beltrán, 1964; Beltrán, 1966.

31 Cabré, 1925; Nony, 1969 fait la synthèse de toutes les hypothèses formulées jusque-là.

32 Beltrán Lloris, 1976: 155-156; corrigé dans Beltrán Lloris, 1990: 223-225; puis dans Beltrán Lloris et al., 1995: 235-238. 
faut voir dans ce groupe statuaire la représentation anonyme d'un notable local du début du $\mathrm{I}^{\mathrm{er}}$ siecle a.C., voire de la fin du II ${ }^{\mathrm{e}}$ siecle a.C. Or, malgré les critiques fréquemment formulées à l'encontre de ses conclusions, W. Trillmich a toujours préféré y deviner un portrait réalisé selon les canons artistiques en vogue, à Rome, à l'époque du second triumvirat ${ }^{33}$. Cette datation, qui ne pouvait bien évidemment pas s'accorder avec celle proposée pour le site depuis les années 1980, prend désormais tout son sens ${ }^{34}$.

$\mathrm{Au}$ final, l'ensemble de ces études récentes permet donc de penser que l'agglomération d'Azaila a été occupée jusqu'aux alentours des années 40 a.C. Elle ne peut donc pas avoir été abandonnée suite à un siège survenu au cours des guerres sertoriennes.

Néanmoins, ces conclusions chronologiques ne permettent pas, à elles seules, de réfuter définitivement l'hypothèse d'un siège fatal à la ville. Si celui-ci a existé, il a très bien pu être plus tardif. On pourrait alors penser à l'éventuelle prise de la ville par les troupes césariennes aux alentours de l'an 49 a.C., peu avant ou peu après la bataille d'Ilerda (Lérida) ${ }^{35}$.

Je pense pourtant que ce siège n'a jamais eu lieu, ou du moins que rien dans les indices archéologiques généralement invoqués ne permet de s'en assurer. On peut en effet proposer une lecture démilitarisée, ou pacifique, de chacune des «évidences» mises au jour.

Il convient tout d'abord de s'interroger sur la nature des défenses urbaines alors en place et sur la résistance que les habitants auraient éventuellement pu opposer aux troupes romaines.

À mon sens, l'efficacité des défenses de l'agglomération paraît finalement assez limitée, et ce malgré leur aspect impressionnant (fig. 2 et 5) ${ }^{36}$. On l'a dit, en effet, seule l'acropole est fortifiée. Elle est entourée d'une muraille à soubassement de pierres surmonté d'une élévation d'adobes ${ }^{37}$. Au sud-est et à l'est, c'est-à-dire sur le côté le plus faible, le rempart est renforcé par un fossé d'environ $150 \mathrm{~m}$ de long. Situé 5 à $9 \mathrm{~m}$ en avant de la muraille, il mesure environ $11 \mathrm{~m}$ de large et 4 à $6 \mathrm{~m}$ de profondeur ${ }^{38}$.

On considère généralement, comme pour confirmer sa puissance, que cette enceinte est composée de deux lignes concentriques de fortification ${ }^{39}$, l'une inférieure, l'autre supérieure. Il me semble pourtant qu'il n'en existe qu'une seule. Elle dessine en fait un tracé de type «à escalade» (fig. 5). À l'ouest de l'acropole, l'enceinte suit en effet l'escarpement du Cabezo et devait se dresser au sommet de celui-ci. Les vestiges actuellement visibles ne sont que ceux du parement de son soubassement. Tel un mur de terrasse, il devait recouvrir l'affleurement rocheux jusqu'à son sommet (fig. 6). À l'est, en revanche, la muraille passe au pied de la colline, légèrement en contrebas. En fait, dans la partie orientale de la colline, les murs que les chercheurs attribuent généralement à la deuxième ligne — «haute»— des murailles me sem-

33 Trillmich, 1990: 48-50; Trillmich, 1997: 136.

34 Cette remarque a déjà été faite par Ribera et Marín, 2004-2005: 435-439.

35 C'était d'ailleurs l'hypothèse traditionnellement formulée jusqu'aux années 1980 (Beltrán Martínez, 1964; Beltrán Martínez, 1966; Beltrán Lloris, 1976).

36 On notera, au passage, l'aspect assez traditionnel de ces défenses, d'influence clairement indigène: acropole fortifiée de petite taille, présence d'un fossé précédé de «pierres plantées», tracé à escalade, absence de tours, absence de rue des remparts et maisons adossées par endroits à l'enceinte, élévation d'adobes sur soubassement de pierres... L'apparence des fortifications d'Azaila nous renvoie plus l'image d'un petit site ibère «classique» que celle d'une agglomération précocement romanisée. C'est pour ces raisons que je doute que la majorité des fortifications de la ville soit, dans leur phase d'aménagement primitif du moins, postérieure au milieu du $\mathrm{II}^{\mathrm{e}}$ siècle a.C.

37 Le soubassement est élevé en blocs de petit appareil irrégulier ou à tendance rectangulaire. Les moellons de gypse et de marne sont posés en assises horizontales. Cabré dit avoir retrouvé, par endroits, quelques assises de briques crues qui les surmontaient (Cabré, 1929: 6).

38 Cabré, 1929. Il s'agit d'un fossé en «U» évasé et à fond plat. Dans sa partie méridionale, il est délimité par un mur d'escarpe, à l'ouest, et par un mur de contrescarpe, à l'est. Plus au nord, seul le mur d'escarpe a été aménagé. Son fond était recouvert d'une couche d'argile mêlée de pierres.

39 Beltrán Lloris, 1976; Asensio, 1995: 146-167; Romeo, 2004. 


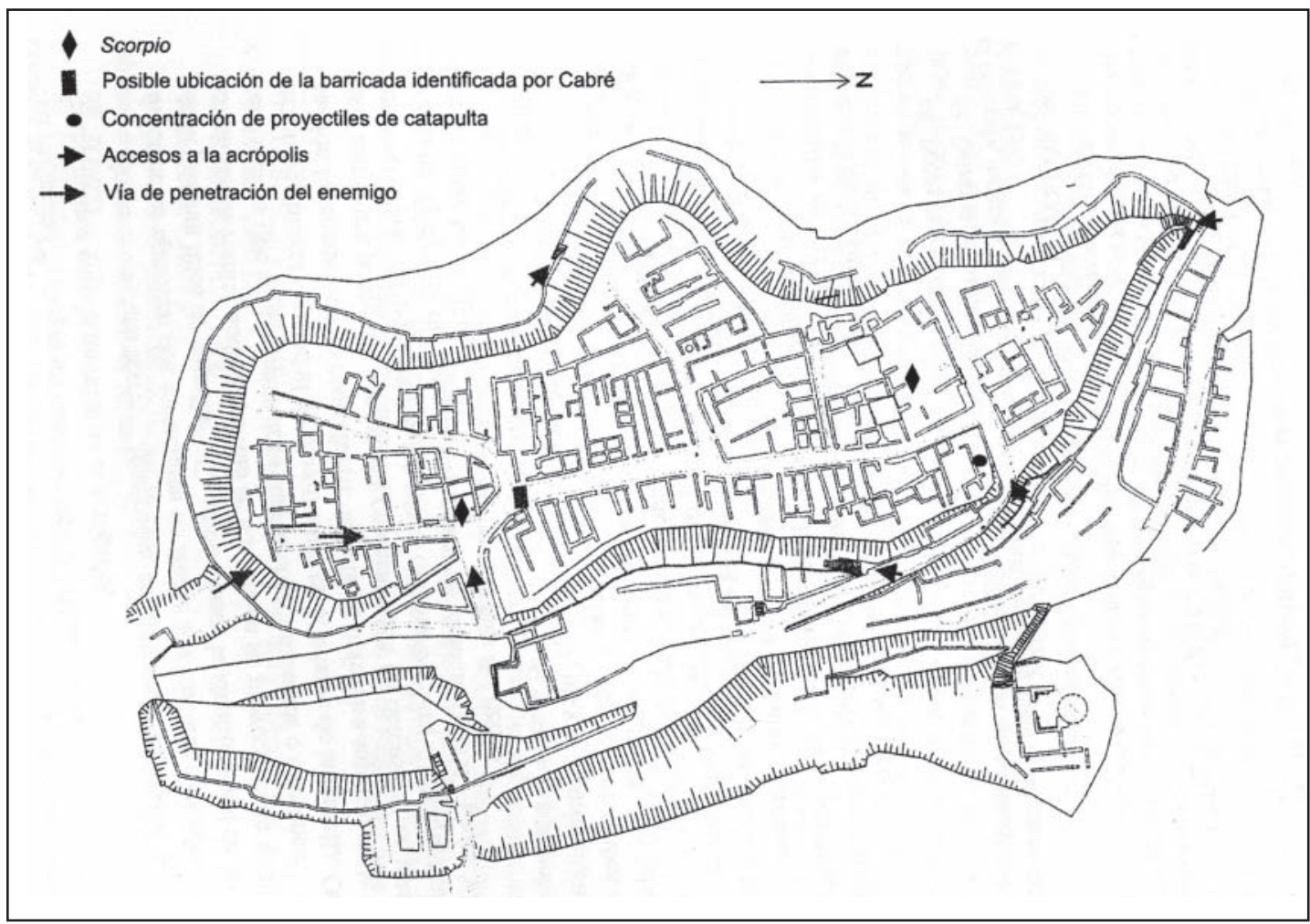

Figure 4. Proposition de déroulement de la prise de l'acropole d'Azaila (d'après Romeo, 2004: fig. 4).

blent plutôt appartenir à des structures de soutènement destinées à organiser les îlots urbains et délimiter les habitats. Ces murs sont clairement indépendants de l'enceinte.

On ne connaît malheureusement pas la hauteur originelle des remparts. Si, du fait de la topographie (fig. 2), on sait que le chemin de ronde de la muraille ouest devait se situer à près d'une vingtaine de mètres au-dessus du sol de circulation extérieur, il est fort probable que, sur le côté oriental, le rempart était beaucoup moins élevé. Cependant, au vu de la largeur de la muraille méridionale ${ }^{40}$, on peut penser que celle-ci atteignait au moins $5 \mathrm{~m}$ de hauteur.

Néanmoins, l'aspect massif de l'enceinte, ainsi que l'impression de bastion inexpugnable qui se dégage à première vue du site, est légèrement nuancé par le grand nombre d'accès à la ville haute qui scandent le tracé de la muraille (fig. 5). Ainsi, on peut restituer au moins huit portes ou poternes sur l'ensemble du petit périmètre fortifié. Elles sont espacées de 40 à $100 \mathrm{~m}$ au maximum. Les trois portes charretières — au sud, au nord et au sud-est— - sont généralement associées à des passages piétons. Ailleurs, des escaliers extérieurs permettaient d'accéder à d'autres poternes. C'est là, sans conteste, l'une des principales originalités d'Azaila. Bien que l'escalier le mieux conservé soit celui qui, à l'est, donne accès aux thermes (fig. 7), le plus spectaculaire demeure celui situé à l'ouest (fig. 8). Même si seules ses neuf premières marches sont conservées, il ne fait aucun doute qu'il devait, à l'origine, conduire à une petite porte

40 La largeur de la muraille est difficile à restituer. Dans la plupart des cas, il ne subsiste en effet que le parement extérieur du mur de soubassement construit selon la technique du «mur de terrasse». Ce parement mesure généralement 0,80 m (Cabré, 1929 et 1944). Néanmoins, au sud, le rempart est visiblement plus épais. Construit selon la technique du «mur simple», il mesure environ $2 \mathrm{~m}$ de large et ne dépasse pas 2,50 m. 
ouverte une dizaine de mètres plus haut. Cet escalier, qui se traduit par un léger renforcement du soubassement de la courtine, se situe dans le prolongement de la «calle D» de l'acropole. En fait, il me semble fort probable que, sur le côté occidental, chacune des rues communiquait avec une poterne qui, elle-même, donnait sur un escalier extérieur (fig. 5) ${ }^{41}$.

On le voit donc, Azaila semble offrir, à première vue et en partie du moins, l'aspect d'un petit bastion fortifié, sorte de nid d'aigle perché sur le sommet d'une colline escarpée et replié sur lui-même. Cependant, il ne fait aucun doute que cette enceinte n'aurait que très difficilement pu arrêter les assauts des armées romaines et de leur machinerie en cas de siège ${ }^{42}$. Il convient en effet de noter que ce rempart n'est flanqué d'aucune tour, à l'exception de celles qui protégeaient vraisemblablement la porte méridionale ${ }^{43}$. De même, selon une conception propre au monde indigène, cette muraille est en grande partie dépourvue de rue des remparts, ou de «boulevards». Cela réduit considérablement les possibilités de déplacement des soldats et des habitants en armes en cas d'attaque. Enfin, il semble que, au moment de l'abandon du site, le fossé avait en grande partie perdu de son rôle défensif. Il servait également, et peut-être même avant tout, de rue. En effet, comme je l'ai dit plus haut, le fond du fossé était recouvert d'une couche de pierres et d'argile. Or, à ce sujet, certains chercheurs n'hésitent pas à parler de «pavement» ${ }^{44}$. Il ne fait donc aucun doute, selon moi, que la circulation y était possible et que, peu avant l'abandon du site, ce fossé servait plus de rue que de moyen de défense ${ }^{45}$. C'est d'ailleurs la conclusion que l'on peut également déduire de la présence des escaliers extérieurs sud-est et est (fig. 5). Puisqu'ils prennent naissance au fond du fossé, ils montrent que son accès était aisé. De plus, le plan suggère que la rue dessinée par le fossé conduit non seulement aux thermes et à la poterne est, mais qu'elle y rejoint aussi une autre rue dallée, d'axe est/ouest (fig. 1). Enfin, il convient de noter que ce fossé semble loin d'être infranchissable. À l'est, il est en effet enjambé par un pont qui relie la «ville basse» à l'acropole par le biais de la porte sud-est ${ }^{46}$.

${ }^{41}$ C'est très certainement le cas de la «calle A», située juste au-dessus de la porte principale méridionale. De même, la «calle B», au sud-ouest, se termine au-dessus de deux renforcements de la muraille. Ils abritaient sans doute un ou plusieurs escaliers. On l'a dit, la «calle D» aboutit, quant à elle, à l'escalier extérieur ouest. Plus au nord, il est fort probable qu'au moins l'une des deux rues parallèles suivantes donnait aussi sur une autre poterne. Enfin, la rue septentrionale conduisait à un escalier qui menait à la porte nord.

42 Les remarques qui suivent ne doivent pas prêter à confusion. L'inadaptation des défenses locales aux techniques de siège romaines ne signifie pas que le siège n'a pas eu lieu, ni que les fortifications de la ville n'ont pas été efficaces. Je cherche seulement à rappeler que, au vu des défenses du site, les troupes italiques n'avaient sans doute pas besoin de réaliser de substantiels travaux de terrassement pour conquérir la ville.

43 Les seules tours généralement mentionnées sont les deux hypothétiques tours de guet situées sur la partie septentrionale de l'acropole (Beltrán Lloris, 1976; Asensio, 1995; Romeo, 2004). Mises au jour par Cabré, elles sont de forme rectangulaire. Distantes d'environ $7 \mathrm{~m}$, elles mesurent environ 4,60 m de côté. Même si ce genre de structures semble fréquent dans la région et pour l'époque (Asensio, 1995), je doute néanmoins de leur rôle militaire et défensif. Le contexte domestique dans lequel ces édifices sont insérés, ainsi que leur forme relativement identique à celle des maisons voisines, me conduisent à y voir, plutôt, les vestiges d'habitats aristocratiques «à tour».

44 Romeo, 2004: 28.

45 Ce genre de situation n'est pas inconnu. Comme me l'a rappelé P. Moret, on retrouve le même cas de figure sur le site d'El Palao (Alcañis, Teruel) (Benavente et al., 2004: 360). Sur ce site, le fossé qui coupe le plateau dans le sens de la largeur a sans doute joué un rôle défensif au cours du Premier Âge du Fer, mais il ne servait plus que de chemin d'accès surcreusé lorsque, dès la fin du II ${ }^{\mathrm{e}}$ siècle a.C. ou au début du siècle suivant, l'agglomération s'est développée sur l'ensemble du plateau.

${ }^{46}$ La présence de ce pont — qui condamne en grande partie la valeur défensive du fossé- me laisse penser que le fossé a été creusé lors d'une phase d'aménagement antérieure. Le pont, la «rue du fossé» et les thermes pourraient n'avoir été aménagés que dans le courant du ${ }^{\text {er }}$ siècle a.C. De plus, contrairement à ce que semble penser Cabré (1929, 1941 et 1944), il me paraît exclu que cet aménagement puisse avoir été un «pont-levis». À ma connaissance, ce genre d'édifice me paraît inconnu dans l'Hispanie préromaine ou de l'époque tardo-républicaine. Il ne devient d'ailleurs fréquent qu'au Moyen Âge. 
On le comprend donc, il est peu probable que, en cas de siège de l'agglomération, ces défenses eussent permis à Azaila de résister longtemps aux assauts des troupes romaines ${ }^{47}$. C'est pour cette raison que, selon la majorité des chercheurs, la communauté aurait cherché à ériger de nouvelles défenses. Les unes en prévision de l'attaque - il s'agit des hypothétiques «pierres plantées»-, les autres en dernier recours contre la progression des troupes ennemies -il s'agirait des «barricades» et des édifices transformés en bastions par l'ajout de pièces d'artillerie.

Le problème posé par les hypothétiques «pierres plantées» ${ }^{48}$ du site d'Azaila est complexe. Il soulève en effet deux séries d'interrogations. La première concerne la nature de cet aménagement, la seconde sa datation.

C'est à M. Beltrán Lloris que revient la paternité de l'interprétation de ces pierres découvertes par J. Cabré49. Jusqu'alors, leur intérêt était passé inaperçu. Il s'agit, en fait, de plusieurs rangées de blocs fichés en terre. Espacées d'environ 0,50 m, les pierres sortent du sol d'environ 0,20 à 0,50 m (fig. 9). Elles ne semblent suivre aucun alignement particulier et l'on devine, tout au plus, une organisation en quinconces. Aujourd'hui disparus, ces blocs ont été mis au jour au début du $\mathrm{XX}^{\mathrm{e}}$ siècle lors de la fouille du grand talus sud-est ${ }^{50}$. On ne connaît néanmoins ni leur localisation précise, ni leur étendue originelle (fig. 3$)^{51}$. On sait seulement que ces rangées de pierres se dressaient en avant du fossé et que leur plus forte concentration formait une bande d'une trentaine de mètres de largeur, située entre 20 et $50 \mathrm{~m}$ en avant de la muraille (fig. 5).

Ces caractéristiques rappellent bien évidemment celles des «pierres plantées» connues dans le monde celtique à l'époque préromaine ${ }^{52}$. Pourtant, cette interprétation est loin d'être assurée. Il faut en effet se souvenir que, lors des fouilles du début du $\mathrm{XX}^{\mathrm{e}}$ siecle, Cabré avait considéré que ces blocs fichés en terre servaient en fait à maintenir et à stabiliser les remblais du talus. Selon lui, elles faisaient partie du processus de construction du «tumulus». C'est une possibilité, mais on verra plus loin que la stratigraphie interne du terre-plein rend, à mon sens, cette interprétation moins plausible que celle proposée par M. Beltrán Lloris ${ }^{53}$.

47 Pour des considérations plus générales sur les techniques poliorcétiques romaines et les sièges voir, par exemple, Sáez Abad, 2003; Campbell, 2005. F. Quesada dresse un bilan de la situation dans l'Hispanie préromaine (Quesada, 2007).

48 Ces alignements de pierres sont ce qu'on appelle couramment, et de façon impropre, des «chevaux de frise». À leur sujet voir Harbison, 1971 et, plus récemment, Alonso et al., 2003. À la suite de P. Moret, je préfère utiliser la formule de «pierres plantées» qui correspond mieux à la nature de ces structures et traduit parfaitement les termes espagnols «piedras hincadas» (Gailledrat et Moret, 2003).

49 Beltrán Lloris et al., 1995: 175.

50 Comme on le verra plus tard, Cabré pensait que ce talus correspondait à un «tumulus ibérique». Depuis l'interprétation proposée par Beltrán Lloris (Beltrán Lloris et al., 1995), on considère qu'il s'agit d'une rampe d'assaut construite par les troupes romaines pour conquérir la ville.

51 Romeo (2004: 27-31) pense que ces pierres bordaient l'ensemble du fossé et qu'elles formaient une bande d'environ $150 \mathrm{~m}$ de long. Cette proposition demeure cependant hypothétique. Rien dans les plans publiés par Cabré, ni dans les photographies prises à l'époque des fouilles, ne le laisse imaginer.

52 Harbison, 1971; Ruiz Zapatero, 2003; Berrocal et Moret, 2007: 21-24. Leur rôle défensif est largement inconnu et leur efficacité paraît somme toute assez limitée. Selon les théories les plus vraisemblables, ces alignements de pierres pourraient avoir servi à ralentir la progression des fantassins ennemis et à les exposer aux flèches des défenseurs. En effet, la bande située entre $15 / 20 \mathrm{~m}$ et $50 \mathrm{~m}$ en avant de la muraille correspond à la distance à partir de laquelle les jets de lance cessent d'être mortels, mais pour laquelle les tirs des archers sont les plus efficaces. Quoi qu'il en soit, il est acquis que ces aménagements servaient contre l'infanterie et non contre la cavalerie (Moret, 1996: 226-227).

53 Il existe une troisième possibilité. Ces pierres pourraient en fait correspondre à des cippes funéraires. En effet, leur position le long de la rue principale et leur forme rappellent certaines nécropoles. L'existence d'un possible «tumulus» funéraire à proximité rend l'hypothèse plus plausible encore. Cependant, cette interprétation se heurte 


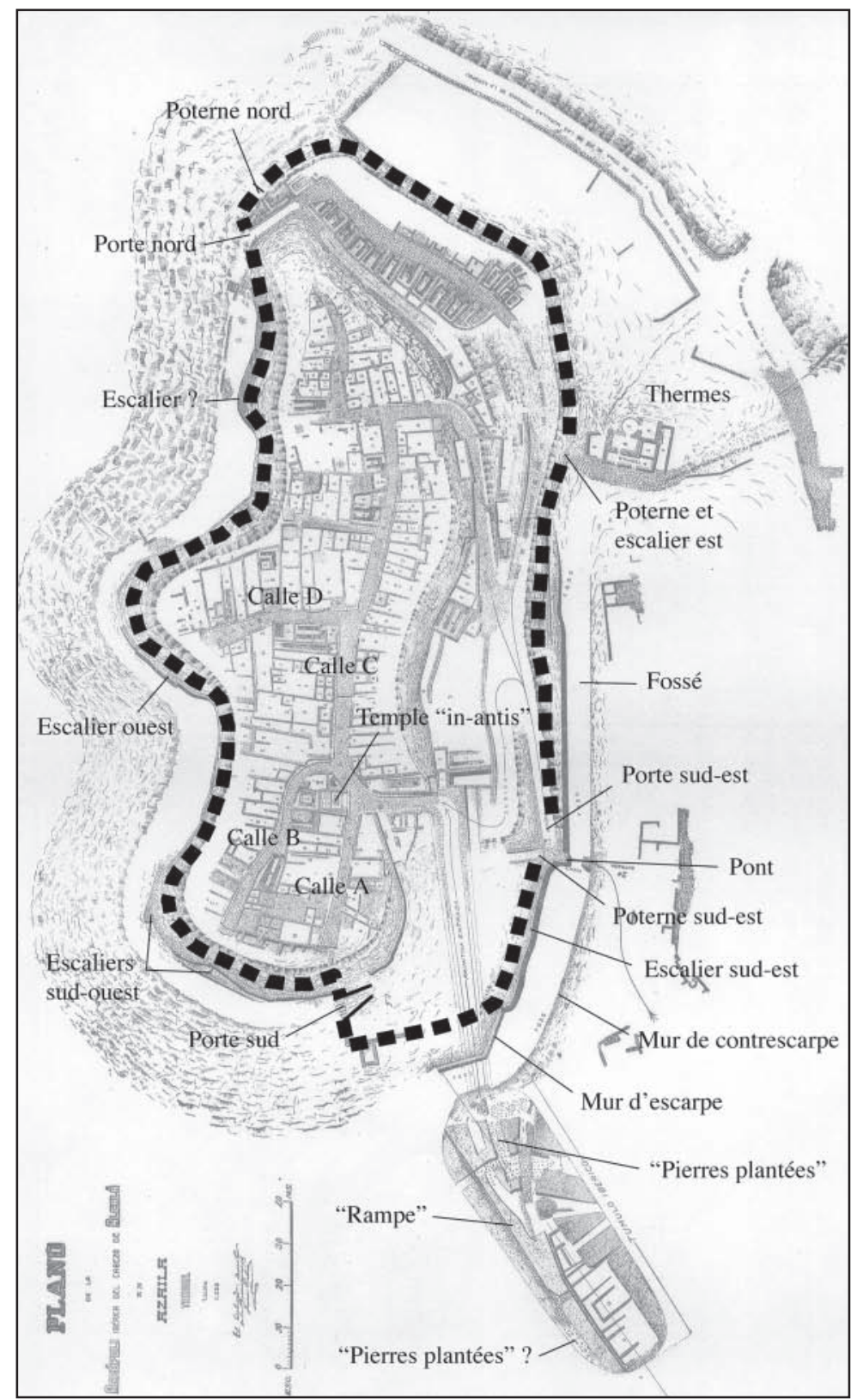

Figure 5. Plan des fortifications de l'acropole d'Azaila (Hourcade d'après Cabré, 1944: fig. 1).

En fait, tant que l'on ne saura pas quelles étaient la position exacte de ces blocs et la superficie sur laquelle ils s'étendaient, leur nature demeurera incertaine. Les plans publiés par Cabré donnent en effet l'impression que certaines de ces pierres ont été découvertes sous la partie sud du talus, à plus de $80 \mathrm{~m}$ de distance du fossé (fig. 1 et 5). Si ces blocs appartiennent

à un écueil de taille. Il n'est nulle part fait mention, dans les notes manuscrites et les publications de Cabré, de la découverte de corps ou d'urnes cinéraires. 
au même système que ceux situés plus au nord, alors il me semble peu probable qu'il s'agisse de «piedras hincadas». Leur dispersion serait trop grande. Si, en revanche, les blocs repérés au sud du terre-plein ne sont que les vestiges des dalles du pavement de la rue principale, alors rien ne s'oppose à ce qu'ils appartiennent au système défensif de la ville. De même, si d'autres blocs venaient à être découverts en dehors de l'emprise du talus, alors l'hypothèse technique formulée par Cabré devrait être définitivement écartée.

Le problème de la datation, relative et absolue, de ces pierres se révèle tout aussi délicat à résoudre que celui de leur nature. Sont-elles, ou non, contemporaines de la construction des fortifications de l'acropole et de l'urbanisation de la «ville basse»?

Là encore, on ne peut que regretter l'absence de fouilles fines et de stratigraphies publiées. Néanmoins, Beltrán Lloris pense pouvoir déduire des plans publiés par Cabré, ainsi que de la documentation inédite, que ces pierres sont postérieures à l'aménagement des rues ${ }^{54}$. Il en conclut qu'elles ont été plantées en vue du siège, pour renforcer les défenses déjà existantes de la ville. Selon lui, en effet, les pierres mises au jour se dressaient en partie au milieu des rues de la ville. Elles remploieraient même certaines des dalles du pavement. Pourtant, il me semble que, sur ce point, il faut une nouvelle fois rester prudent. Le plan me paraît plutôt indiquer que les «pierres plantées» sont, au contraire, implantées en bordure des rues et à l'extérieur des maisons, et non à l'intérieur de celles-ci (fig. 1). Elles me semblent donc respecter l'urbanisme en place.

Un autre argument me laisse d'ailleurs penser que ces «obstacles» n'ont pas endommagé les structures urbaines antérieures. C'est celui de leur efficacité. Le plan montre en effet que la rue principale mise au jour sous le talus était, en grande partie, restée praticable. Or, dans ces conditions, on comprend mal pourquoi les habitants de la ville auraient cherché à ralentir la progression des troupes ennemies par l'ajout de quelques «pierres plantées», mais les auraient laissé emprunter librement les rues qui conduisent à la ville haute. De plus, l'arasement total des maisons — préalable supposé à l'installation des «piedras hincadas»— aurait, à mon avis, constitué une erreur tactique. On pourrait certes y voir la preuve de la détermination des habitants d'Azaila, prêts à sacrifier certains quartiers mal défendables pour assurer une meilleure protection de l'acropole. Mais c'est oublier que ces travaux auraient non seulement monopolisé longuement la main d'œuvre locale, mais aussi que la communauté se serait ainsi privée d'obstacles déjà existants. Pour ralentir la progression des troupes, il aurait sans doute été plus rapide et judicieux de détruire les maisons et d'en laisser une partie des décombres sur place. Ceux-ci auraient été tout aussi efficaces, voire davantage, que les «pierres plantées» ${ }^{55}$.

Au final, on le voit, le dossier est complexe. Néanmoins, je serais enclin à penser que, si ces blocs appartiennent au système de défense de l'agglomération, alors ils sont contemporains, voire antérieurs, à l'urbanisation de ce quartier de la ville basse. Cela ne doit d'ailleurs pas surprendre. Dans la quasi-totalité des cas connus, la mise en place des fortifications accompagne en effet le processus de fondation et/ou d'urbanisation de l'agglomération. Sauf modifications et ajouts très ponctuels, il n'existe aucun cas avéré de défenses urbaines en pierres construites en temps de guerre pour faire face à un siège ${ }^{56}$. $\mathrm{Si}$, au contraire, ces pierres sont postérieures

54 Beltrán Lloris et al., 1995: 175.

55 Même si la fonction de ces obstacles reste débattue, la majorité des chercheurs s'accorde à penser que leur rôle défensif était relativement assez limité. On insiste désormais plus sur leur aspect symbolique et monumental (Alonso et al., 2003).

56 La construction des fortifications appartient à un autre temps que celui du conflit. Élevées lors de la (re) fondation de la ville, elles servent à se prémunir contre des périls supposés ou fantasmés, en prévision des guerres redoutées. Non au moment, ou après, le déclenchement de celles-ci. La relecture critique des découvertes faites sur les sites d'Osuna (Séville) ou de Libisosa (Lezuza, Albacete), par exemple, me paraît en ce sens révélatrice (Hourcade, 2003; Hourcade, à paraître). 


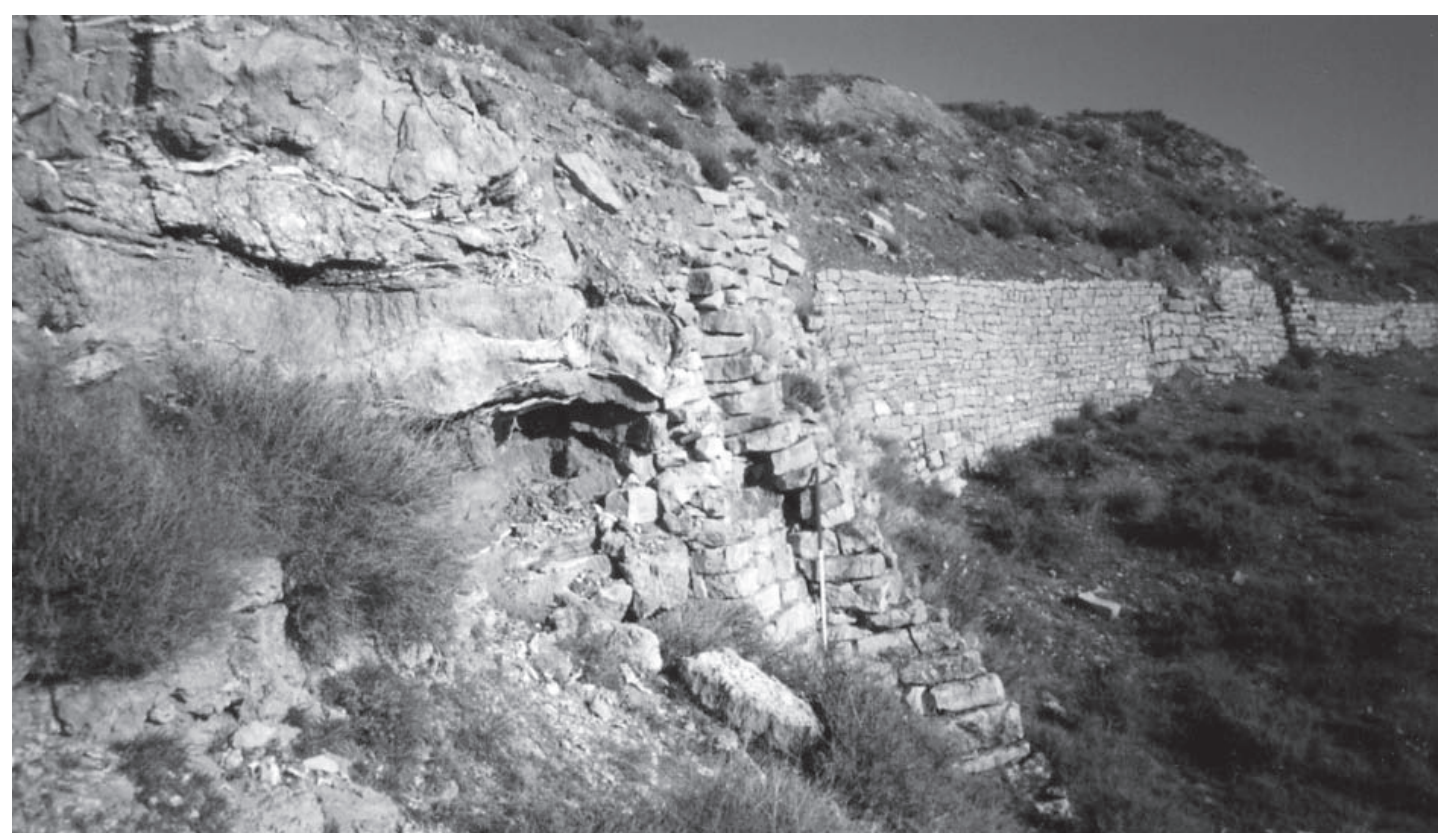

Figure 6. Parement extérieur du soubassement de la muraille ouest d'Azaila (cl. D. Hourcade).

aux maisons, alors elles ne jouent probablement aucun rôle défensif ${ }^{57}$. Dans tous les cas, on le voit, ces blocs ne me semblent pas devoir, ni pouvoir, être perçus comme les témoins d'un siège que la ville aurait subi ${ }^{58}$.

Les autres défenses, que les habitants de l'agglomération auraient élevées, correspondent aux amoncellements que Cabré avait interprétés comme des «barricades». Elles auraient été installées pendant le combat, une fois le rempart franchi par les troupes romaines, pour tenter de ralentir leur progression dans la ville haute (fig. 4).

On ne sait malheureusement presque rien de ces aménagements. Deux d'entre eux auraient été repérés en travers des rues $\mathrm{A}$ et $\mathrm{C}^{59}$. S'il ne fait aucun doute que les ouvriers de Cabré ont effectivement mis au jour des amoncellements de pierres, je suis en revanche plus sceptique en ce qui concerne leur interprétation. Là encore, il me semble qu'il faille faire preuve de la plus grande prudence.

57 Comme je l'ai dit plus haut, c'est l'hypothèse formulée par Cabré. Je reviens sur ce point plus loin, lors de l'analyse de la «rampe d'assaut».

58 Cela poserait d'ailleurs un autre problème d'ordre chronologique. Si ces hypothétiques «pierres plantées» dataient d'un siège survenu dans le courant des années 40 a.C. - comme nous l'avons vu supra, on doit en effet abandonner l'hypothèse d'une datation du premier tiers du ${ }^{\text {er }}$ siècle a.C. puisque les nouvelles études céramologiques montrent que le site est occupé jusqu'aux années 50/30 a.C.-- alors il s'agirait de l'exemple le plus tardif attesté pour la région. En effet, dans cette partie nord et est de la Meseta, tous les autres cas connus datent du Premier $\hat{A} g e$ du Fer ou du début du Second Âge du Fer. Ils sont tous antérieurs à la conquête romaine. On connaît certes des exemples d'utilisation de «pierres plantées» plus tardifs —il en existe en fait des attestations jusqu'au début du Haut-Empire-, mais ils appartiennent à un domaine géographique et culturel bien différent. C'est celui des villages du nord-ouest de la péninsule (Moret, 1996: 223-227; Berrocal et Moret, 2007: 21-24). Si, en revanche, ces défenses sont contemporaines des autres fortifications de la ville, elles pourraient plutôt dater des $\mathrm{III}^{\mathrm{e}}$ ou $\mathrm{II}^{\mathrm{e}}$ siècle a.C. Leur présence sur le site serait alors moins exceptionnelle.

59 Leur position permet d'ailleurs à F. Romeo d'essayer de restituer le déroulement du siège et les étapes de la progression des troupes ennemies dans la ville (Romeo, 2004). 
La première raison qui me fait douter de l'interprétation proposée tient au fait que, par nature, les barricades sont des édifices temporaires. Elles ne résistent généralement que peu aux assauts du temps. On imagine donc mal — même si le site a été rapidement abandonné par la suite - comment elles auraient pu traverser les siècles intactes. De plus, une fois la ville prise, leur position aurait rendu l'évacuation de l'acropole et des habitats assez difficile ${ }^{60}$.

La seconde raison est due à l'absence de stratigraphie et de descriptions détaillées. On ne peut en effet s'empêcher de penser que ces amoncellements de pierres peuvent, en fait, provenir de l'effondrement de structures en élévation. Dans ces conditions, leur position, en travers des rues antiques, pourrait n'être que fortuite. Rien ne permet non plus de certifier que ces structures sont antiques. En fait, elles me font penser à des pierriers postérieurs à l'abandon du site. Ainsi, on pourrait tout aussi bien imaginer que ces amoncellements de blocs ont été aménagés à partir de matériaux récupérés dans les décombres. On sait en effet qu'à l'époque moderne, mais peut-être aussi dès l'Antiquité, le site a servi de carrière d'extraction de matériaux lors de la construction du bourg voisin ${ }^{61}$. On imagine alors volontiers que les équipes chargées du terrassement constituaient, par commodité et à intervalle régulier, des tas de pierres que les charrettes venaient récupérer par la suite. Certains n'auraient finalement pas été évacués. Il se pourrait aussi qu'il s'agisse de «rebuts» abandonnés sur place au profit de blocs de meilleure qualité.

Bien que cette hypothèse demeure malheureusement invérifiable, elle montre néanmoins que l'on peut apporter une lecture civile à la présence de ces supposées «barricades». Leur existence ne peut donc pas être perçue comme la preuve indéniable d'un conflit sur le site ${ }^{62}$.

On peut d'ailleurs faire le même type de remarques au sujet de l'ensemble des armes —catapultes, armes de poing et de jet- mises au jour sur le site lors des fouilles de Cabré6 ${ }^{3}$. Contrairement à ce que l'on pense généralement, elles ne me semblent pouvoir prouver ni qu'il y a eu conflit — du moins pas sur la colline—- ni qu'il s'agissait d'un siège ${ }^{64}$.

Les armes les plus célèbres découvertes à Azaila sont deux catapultes de torsion de type scorpio $^{65}$. Leurs restes ont été mis au jour en 1925, dans le temple dit «in antis», pour la première, et en 1942 dans une «maison de chef», pour la seconde (fig. 4) ${ }^{66}$. La plupart des chercheurs considèrent qu'elles témoigneraient de la lutte acharnée des habitants de la ville lors du siège de l'acropole. Ainsi, leurs lieux de découverte correspondraient aux ultimes bastions que les défenseurs auraient aménagés dans l'urgence afin de tenter d'enrayer l'avancée de l'armée

60 L'absence de certains indices archéologiques permet en effet de penser que la ville a été évacuée après que l'on ait récupéré la plupart des objets utiles ou de valeur. Ainsi, lors de la fouille des habitats, les ouvriers n'ont, par exemple, pas trouvé trace d'un abondant mobilier quotidien abandonné et écrasé sur place. Ceci implique que les habitants ou les pillards avaient effectué de nombreux allers et venues pour les récupérer avant l'effondrement des structures. Or, il est vraisemblable que cette phase de pillage ou d'évacuation ait nécessité l'emploi de charrettes. Nul doute que les éventuelles barricades aient, à ce moment, été démantelées pour permettre une circulation plus facile dans la ville.

61 Cabré, 1944; Beltrán Lloris, 1976; Asensio, 1995.

62 De plus, comme on le verra plus loin, cette hypothèse pratique et pacifique s'accorde assez bien avec l'interprétation que je propose pour la prétendue «rampe d'assaut» située au sud de l'acropole.

63 On en trouvera la liste dans Beltrán Lloris, 1976 ou dans Saéz Abad, 2006.

64 La présence d'armes sur un site urbain d'époque républicaine est souvent perçue, dans la péninsule Ibérique, comme la preuve d'un siège. Pourtant, mis à part des exemples extrêmement rares, comme celui de Valence (Ribera et Calvo, 1995), c'est rarement le cas (Hourcade, à paraître).

65 Sur les caractéristiques de ce type d'armes voir Richmond, 1945; Marsden, 1969 et 1971; Campbell, 2003a et 2003b; Saéz Abad, 2005.

66 Cabré, 1944; Beltrán Lloris et al., 1995; García Díez, 2002. 


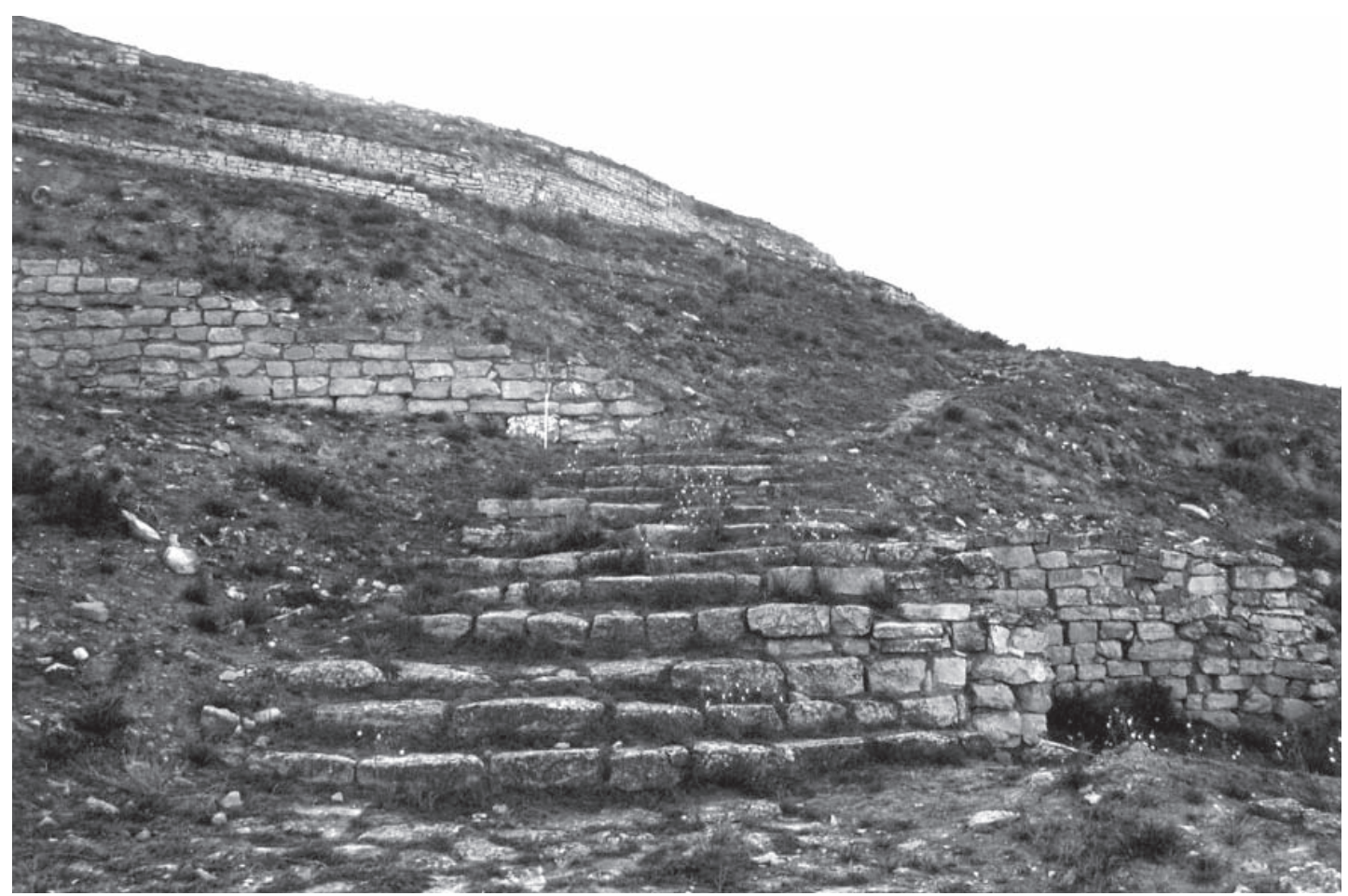

Figure 7. Escalier extérieur de la «poterne est» d'Azaila (cl. D. Hourcade).

ennemie dans la ville ${ }^{67}$. Aussi séduisante et, disons-le, émouvante que soit cette hypothèse, elle s'accorde pourtant mal avec les restes découverts.

En effet, les travaux réalisés par García-Díez sur ces deux pièces d'artillerie ont montré que, au moins en ce qui concerne celle mise au jour dans le temple, elles ne pouvaient pas avoir servi à défendre la ville lors du supposé siège qui aurait conduit à la destruction de celle-ci ${ }^{68}$. La nature des restes, ainsi que leur position lors de leur découverte, prouve non seulement que cette catapulte était déjà démontée au moment de son abandon, mais également que, à l'origine, elle était très certainement appuyée contre le mur ou accrochée à celui-ci. On doit donc en déduire que la catapulte découverte dans le temple était non seulement inutilisable, mais qu'elle avait aussi été déposée, ou plutôt «exposée», à cet endroit. En fait, tout indique qu'il s'agissait non plus d'une arme fonctionnelle, mais très certainement d'un trophée, c'està-dire d'un objet consacré et intentionnellement déposé —une fois démilitarisé- dans un lieu de culte ${ }^{69}$. Sa présence à cet endroit n'a donc rien à voir avec la transformation du temple dit «in antis» en bastion. Elle est le témoignage d'un geste rituel et d'actions militaires passées. De même, bien que les informations manquent à son sujet, il est fort probable que l'autre scorpio ait été également un trophée. Tel un butin de guerre, cette deuxième catapulte aurait, cette foisci, était exposée dans la domus d'un des notables de la ville, non dans un édifice public ${ }^{70}$.

${ }^{67}$ En dernier lieu, voir Romeo, 2004, Saéz Abad, 2005 et Saéz Abad, 2006.

68 García Díez, 2002.

69 Ce genre de pratiques, bien connu dans le monde hellénistique, l'est aussi dans la péninsule Ibérique (Rovira, 1999; Gabaldón, 2004).

70 Même si les trophées militaires exposés en milieu domestique sont le plus souvent des armes individuelles, ce type de cas de figure n'est pas inconnu. On pourra, à ce sujet, relire les hypothèses formulées par les archéolo- 


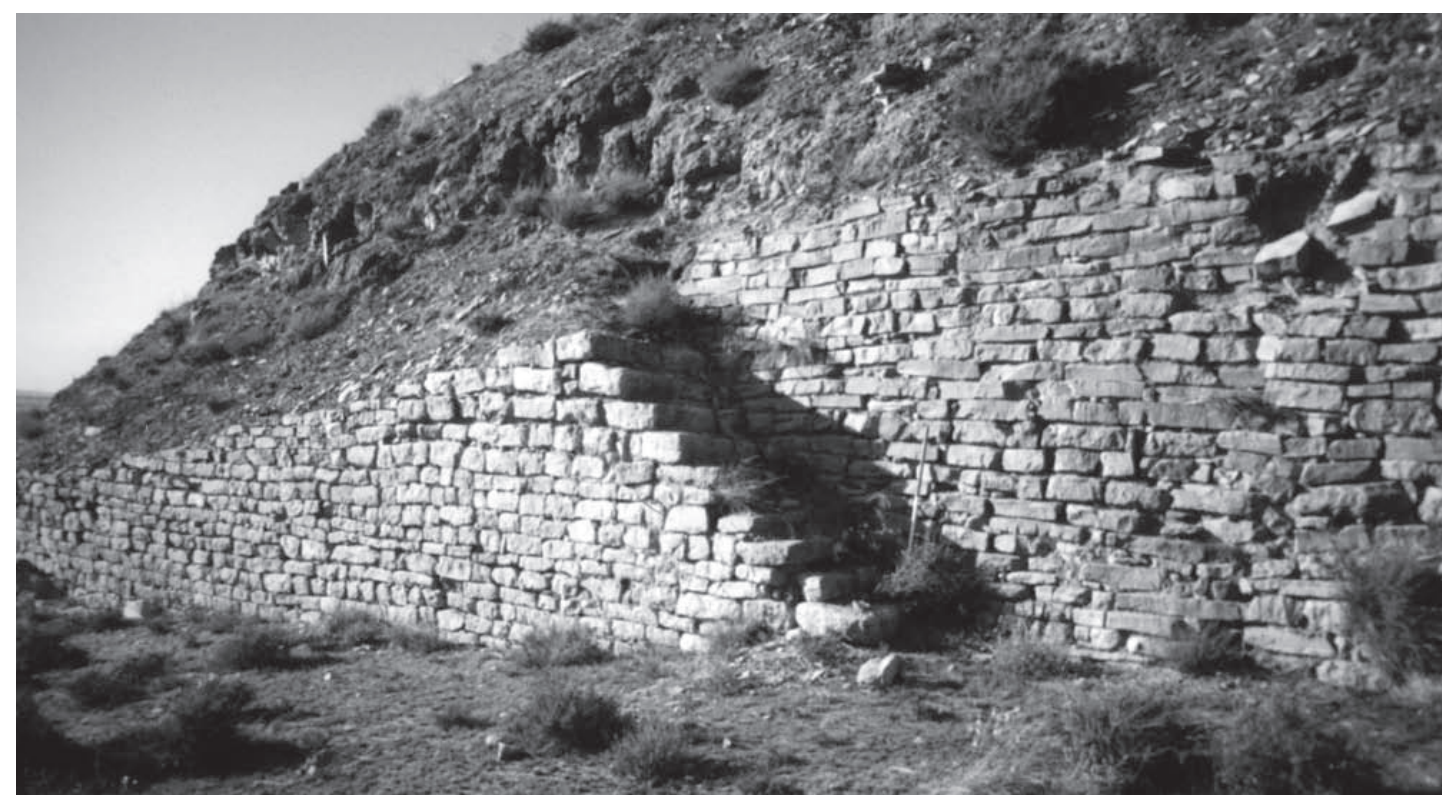

Figure 8. Escalier extérieur et soubassement de la muraille ouest d'Azaila (cl. D. Hourcade).

Il me semble d'ailleurs que cette interprétation permet aussi d'expliquer différemment la présence des quelques autres armes de poing découvertes sur le site. Comme pour les catapultes, on considère parfois que les épées et le poignard mis au jour lors des fouilles de Cabré71 témoignent de la bataille consécutive au siège de la ville. En effet, puisque la plupart de ces armes proviennent du temple, on a tendance à les associer à l'arsenal défensif de cet hypothétique bastion improvisé. Or, il me semble plus probable que, là encore, il s'agissait en fait de trophées, exposés et déposés en offrande ${ }^{72}$. De même, on ne peut pas exclure l'hypothèse que certaines des autres armes de poing, dont le lieu de découverte reste incertain, aient été utilisées en contexte funéraire ${ }^{73}$.

Au final, on le voit, il me semble que, contrairement aux opinions les plus fréquentes, ces armes ne permettent ni de conclure à l'existence d'un siège qui aurait été fatal à la ville, ni à celle d'un combat acharné dans ses rues. Les lieux de découverte des pièces d'artillerie ${ }^{74}$ et de

gues au sujet des nombreuses armes mises au jour autour du patio de la «Casa de Likinete» sur le site de La Caridad (Caminreal, Teruel) (Vicente et al., 1997: 196). Selon eux, il n'est pas impossible qu'il se soit agi de trophées et de «souvenirs» abandonnés sur place par leur propriétaire et non de témoignages d'un siège.

71 Beltrán Lloris, 1976: 175-176.

72 Les données précises manquent au sujet de ces épées. Il semble pourtant que leurs lames étaient perforées. Si tel était le cas, leur fonction ne ferait aucun doute. Ces trous permettaient en effet de les accrocher aux murs des pièces dans lesquelles elles étaient exposées (Rovira, 1999).

73 C'est le cas de celles découvertes à proximité de ce que Cabré nomme le «tumulus ibérique».

$74 \mathrm{Il}$ est, à ce sujet, intéressant de noter qu'aucune des quatre catapultes de torsion de type scorpio découvertes dans la péninsule Ibérique ne témoigne de façon certaine d'un siège ou d'un conflit survenu sur le lieu de leur découverte (Hourcade, à paraître). Ainsi, la catapulte de la «Neapolis» d'Emporion (Ampurias, Gérone), bien que généralement considérée comme datant du siège de la ville par Caton (Bosh Gimpera, 1913-1914; Saéz Abad, 2006), me semble en fait appartenir à l'arsenal de l'agglomération impériale. De même, comme je l'ai déjà rappelé, celle mise au jour dans la «Casa de Likinete» à La Caridad (Caminreal, Teruel) pourrait n'être qu'un trophée (Vicente et al., 1997). 
certaines épées ne correspondent donc sans doute pas aux derniers retranchements des défenseurs, mais plus simplement à leurs lieux de dépôts et de consécration.

Néanmoins, il est pourtant certain que ces militaria témoignent d'un ou de plusieurs conflits armés $^{75}$. Le problème reste que celui-ci (ou ceux-ci) nous demeure(nt) totalement inconnu(s). On ne peut savoir ni où, ni quand, ni par qui, ces catapultes et ces armes de poing ont été utilisées. En effet, leurs caractéristiques ne permettent pas de les dater avec précision ${ }^{76}$ et il est impossible de savoir si elles ont servi à défendre la ville lors d'un conflit antérieur ${ }^{77}$ ou si, au contraire, elles ont été prises, à une armée ennemie et sur un autre site, par quelques soldats ou guerriers vivant à Azaila.

De plus, c'est, le même type de problèmes - concernant le rôle et la datation de ces objets - qui se pose au sujet des autres armes de jet mises au jour sur le site. On compte en effet plusieurs pointes et talons de lances, des flèches, quelques balles de fronde, ainsi qu'un lot important de boulets de catapultes (fig. 4) ${ }^{78}$. Comme les armes précédentes, celles-ci sont généralement utilisées comme argument pour témoigner de l'âpreté des combats survenus lors du siège. Or, là encore, il me semble que l'on peut trouver une explication différente à leur présence.

Dans la mesure où ces objets ne peuvent pas être datés avec précision, on ne peut pas savoir de quelle époque ils témoignent, ni à quel moment ils ont été utilisés, ni d'ailleurs pour quelle raison. Objectivement, rien ne permet donc de penser qu'ils proviennent obligatoirement d'un siège fatal à la ville.

Pire, on ne sait ni quel était leur rôle, ni qui étaient leurs «propriétaires». En effet, dans la mesure où ces projectiles étaient anépigraphes ${ }^{79}$, il est impossible de savoir s'ils ont été lancés contre les remparts de la ville par une ou plusieurs armée(s) ennemie(s) ou si, au contraire, ils étaient destinés à la défense de la communauté. En d'autres termes, leur présence pose non seulement le problème de la réalité d'un conflit sur le site d'Azaila, mais aussi celui de l'existence d'un arsenal municipal. Les communautés indigènes de la fin de l'époque républicaine en étaient-elles pourvues ? La question est débattue pour l'époque préromaine et le

75 Le fait qu'elles étaient en partie endommagées et démontées montre sans ambiguïté qu'elles ont servi lors d'un conflit, mais aussi qu'elles ont été déplacées par la suite.

76 L'étude de García Díez (2002) montre que ces machines devaient envoyer des flèches d'une longueur approximative de deux coudes (entre 0,846 et $0,90 \mathrm{~m}$ ) et d'un diamètre de 2,6 à $2,8 \mathrm{~cm}$. Ces données ne permettent cependant de tirer aucun enseignement d'ordre chronologique. Le tableau comparatif de toutes les catapultes de type scorpio datées de la fin de la République et du Haut-Empire découvertes dans l'ensemble du monde romain montre en effet que la forme et les caractéristiques de ces machines n'ont que très peu évolué au cours des siècles (Vicente et al., 1997: 181). Ainsi, rien ne permet de faire la différence entre une arme de la fin du II ${ }^{\mathrm{e}}$ siècle, une autre du premier tiers du Ir siècle a.C. ou une troisième des années 40 a.C.

77 Cette question est bien évidemment liée à celle, largement débattue, de la capacité technologique des agglomérations indigènes à se défendre contre les armées extra-péninsulaires. J'évoque ce thème plus loin. Pour une synthèse critique, voir en dernier lieu Quesada (2007).

78 Il semble que l'on ait repéré deux zones de concentration de projectiles en pierre. L'une, très hypothétique, près de la «poterne est» (Cabré, 1929: 9) et l'autre sur le sommet de l'acropole, à l'extrémité nord de la «calle C» (Romeo, 2004). Douze pétroboles sont encore conservés sur le site (Beltrán Lloris, 1976: 175-176). Taillés dans l'albâtre, ils mesurent entre 12,4 et 19,4 cm de diamètre et pèsent entre 1,950 et 7,30 kg. On peut en fait les classer en deux grands groupes. Celui des projectiles pesant environ $3 \mathrm{~kg}$ et celui des boulets d'environ $5 \mathrm{~kg}$. Bien que les plus petits pouvaient être lancés manuellement, il est fort probable qu'ils étaient en fait destinés à l'être à l'aide de catapultes.

79 On connaît quelques exemples de projectiles inscrits. Lorsque leurs textes sont compréhensibles, ils favorisent bien évidemment la datation des armes. C'est le cas, par exemple, de l'un des boulets mis au jour sur le site de Calahorra (La Rioja) (Cinca et al., 2003) et de plusieurs balles de fronde en plomb découvertes en Andalousie ou dans la Péninsule (Díaz Ariño, 2005; Pina Polo et Zanier, 2006). 
début de la conquête romaine ${ }^{80}$. Néanmoins, il me semble qu'à partir du début du Ir $^{\text {r }}$ siècle a.C. au plus tard, la majorité des cités indigènes, sinon toutes, devaient très certainement disposer d'entrepôts d'armes, ainsi que d'un éventail plus ou moins large de pièces d'artillerie légères ${ }^{81}$. On sait d'ailleurs que, même sous le Haut-Empire, c'est-à-dire durant la pax romana, chaque colonie romaine devait être capable d'assurer sa propre protection ${ }^{82}$. La présence d'armes dans la cité était donc une obligation légale, que l'on se trouve ou non en temps de guerre. On voit mal, dans ces conditions, pourquoi cela ne serait pas le cas des communautés pérégrines de la fin de la République, censées assurer par elles-mêmes leur défense ${ }^{83}$.

Pour résumer, on doit donc reconnaître qu'aucune des armes découvertes sur le site d'Azaila, ni qu'aucune des défenses élevées par sa communauté, ne permet de conclure définitivement à l'existence d'un siège qui aurait été fatal à la ville. En effet, pour chaque argument avancé, on peut proposer une autre lecture des vestiges, pacifique et démilitarisée ${ }^{84}$, ou bien civique ${ }^{85}$.

Dans ces conditions, que faut-il penser de la supposée rampe d'assaut mise au jour au sud de l'acropole et qui fait la renommée d'Azaila (fig. 1 à 3, 9 à 11) ? Si les armes et les défenses découvertes sur le site ne peuvent plus être considérées comme des témoins fiables du siège, comment pourrait-il en être autrement de cette «rampe»?

On l'a dit, la majorité des chercheurs pense désormais que ce talus est un agger qui a été élevé par les troupes romaines pour faciliter la prise de la ville en permettant à leurs machines de siège de s'approcher au plus près des remparts ${ }^{86}$. Pourtant, cette hypothèse est relativement récente. En effet, à la suite de Cabré et jusqu'aux années 1970, on interprétait la «rampe» comme un «tumulus» funéraire d'époque ibérique et on pensait que les "pierres plantées» mises au jour dans sa partie basse servaient à en stabiliser les remblais ${ }^{87}$. On le voit donc, pour l'inventeur du site comme pour les chercheurs qui lui ont succédé, les caractéristiques militaires de ce talus semblaient, dans un premier temps du moins, loin d'être évidentes. Elles ne le sont d'ailleurs pas plus pour moi. Les raisons qui me font douter de l'interprétation militaire de ce remblai tiennent à ses caractéristiques techniques: à son mode de construction d'abord, à sa forme ensuite.

80 Deux conceptions s'affrontent au sujet du degré de sophistication tactique des défenses des communautés indigènes avant le I ${ }^{\mathrm{er}}$ siècle a.C. P. Moret et F. Quesada soutiennent un point de vue «minimaliste» qu'il est à mon avis plus sage de préférer (Moret, 1996: 237-263; Moret, 2001; Quesada, 2001; Quesada, 2007). F. Gracia Alonso propose, au contraire, une vision plus «moderne» (Gracia Alonso, 1997; Gracia Alonso, 2000; Gracia Alonso, 2001; Gracia Alonso, 2003; Gracia Alonso, 2006).

81 Voir ce qu'en dit Vitruve, X, 10, 1.

82 C'est ce que nous apprend la Lex Ursonensis, rubrique CIII.

83 Le site d'Arcóbriga (Cerro Villar, Monreal de Ariza, Saragosse) permet d'illustrer cette problématique (Hourcade, à paraître). Dans une des structures du sommet de l'acropole, on a en effet anciennement mis au jour un lot de vingt-trois boulets. Le Marquis de Cerralbo et son commentateur y voyaient la trace d'un siège datant de l'époque républicaine (Beltrán Lloris, 1987). Pourtant, je crois que rien n'interdit d'y reconnaître plutôt les vestiges de l'arsenal de l'agglomération celtibère. Ces projectiles pourraient tout aussi bien dater de la fin de la République que du début de l'Empire.

84 C'est le cas des fausses «barricades», des catapultes et des armes de poing exposées comme trophées.

85 C'est le cas des probables «pierres plantées» et des armes de jet. Elles pourraient en fait appartenir au système de défense de la communauté et à son arsenal, c'est-à-dire à des structures conçues lors de la fondation de la ville et qui n'impliquent pas la réalité d'un conflit.

86 Beltrán Lloris, 1976; Belrán Lloris et al., 1995; Romeo, 2004; Saéz Abad, 2005.

87 Cabré, 1929 et 1944; Beltrán Lloris et al., 1995: 175. 

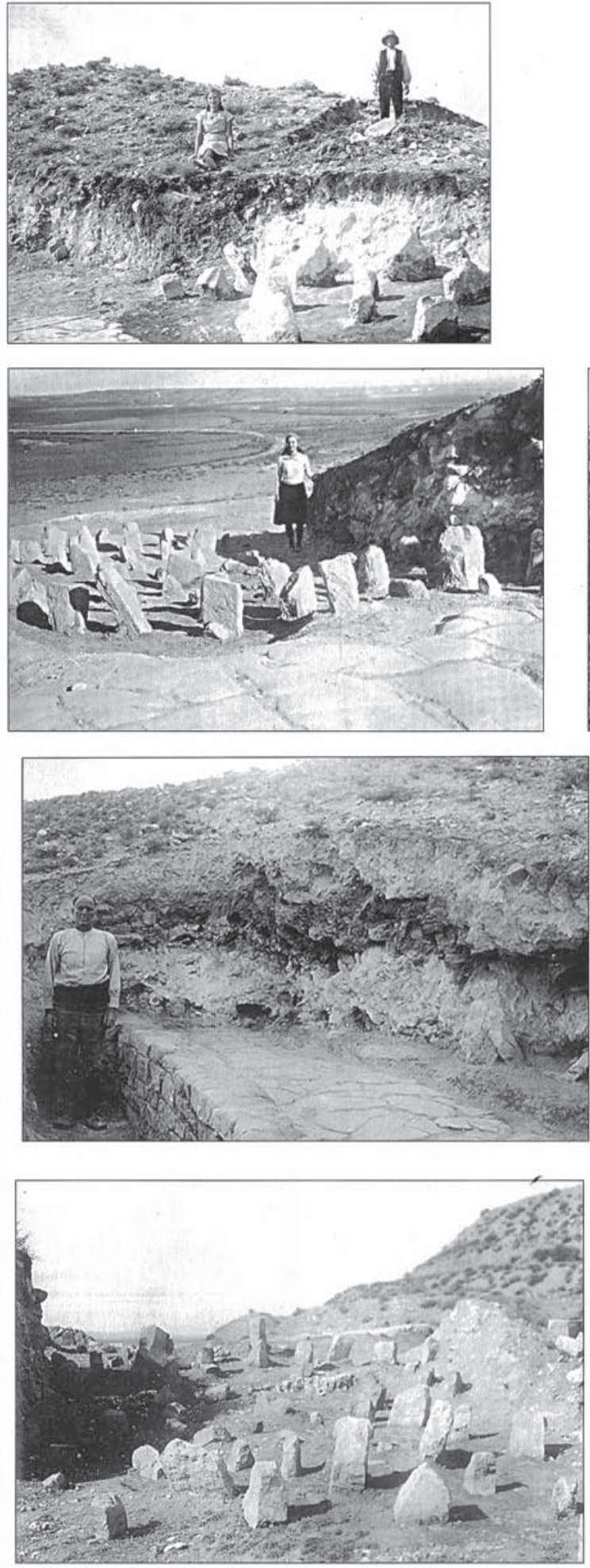

Figure 9. «Pierres plantées» découvertes sous la «rampe» d'Azaila (cl. J. Cabré dans Beltrán Lloris et al., 1995: fig. 45 à 47).
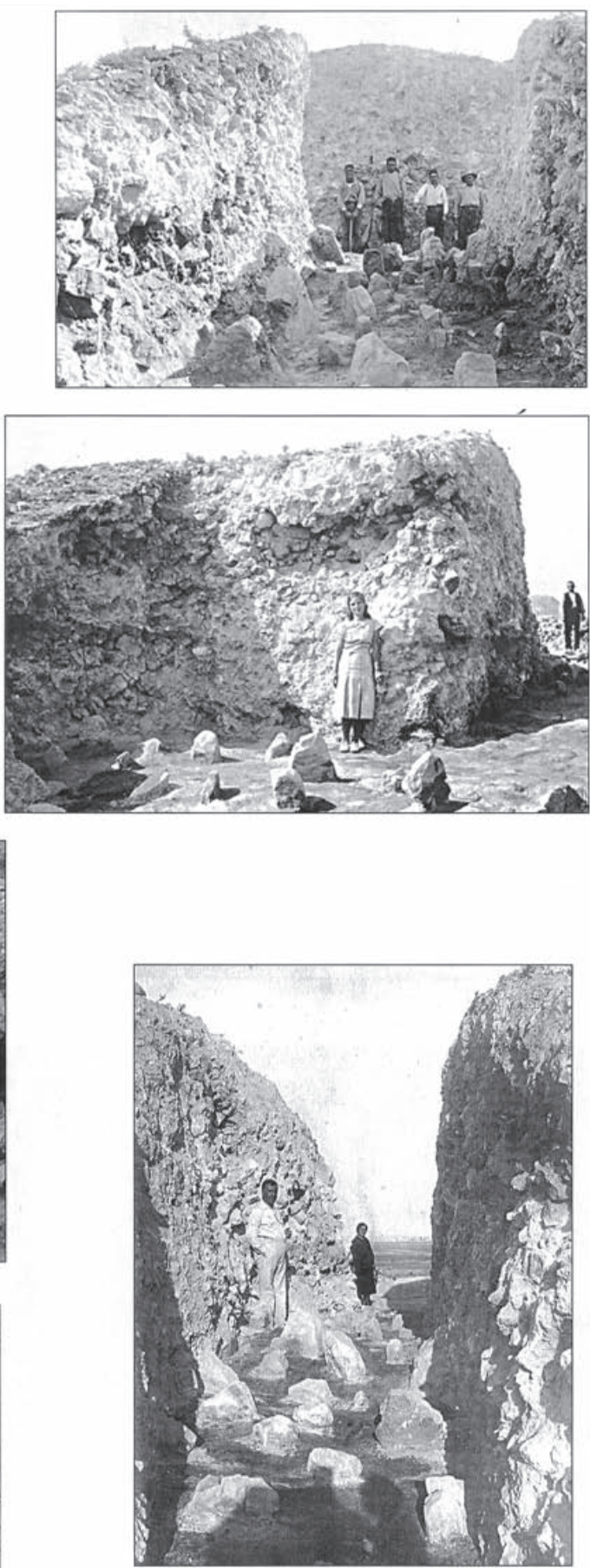

Gladius, XXIX (2009), pp. 93-120. ISSN: 0436-029X 
La nature du remblai est en effet surprenante ${ }^{88}$. Selon Cabré, il s'agit d'une maçonnerie faite de «cal y canto» ${ }^{89}$ et les chercheurs modernes n'hésitent d'ailleurs pas à parler à son sujet d'opus caementicium (fig. 10) ${ }^{90}$. Même si ce terme est peut-être abusif ${ }^{91}$, il pose un double problème, à la fois chronologique et fonctionnel. On sait, en effet, que la technique de l'opus caementicium est connue des Romains depuis, au moins, la seconde moitié du II siècle a.C. ${ }^{92}$ Cependant, son usage dans la péninsule Ibérique est plus tardif. Parfois employé dans les fortifications urbaines dès le début du ${ }^{\text {er }}$ siècle a.C. ${ }^{93}$, il ne devient fréquemment utilisé qu'à partir de l'époque augustéenne, voire après celle-ci ${ }^{94}$. La rampe d'Azaila compterait donc parmi les exemples les plus précoces d'utilisation de ce procédé en Hispanie. Mais le principal problème réside surtout dans le fait que l'emploi de cette technique par une armée en campagne —qui plus est lors d'un combat et d'un siège - me semble particulièrement surprenante, voire incongrue. À ma connaissance, il n'est nulle part fait mention d'une telle pratique par les troupes romaines. Surtout à l'époque républicaine. Généralement, les matériaux employés pour les travaux de terrassement militaires sont la terre, le bois et, dans une moindre mesure, la pierre ${ }^{95}$. De plus, les sources littéraires ${ }^{96}$ et les vestiges archéologiques ${ }^{97}$ ne laissent aucun doute sur la façon habituelle de construire les rampes d'assaut. Elles sont exclusivement aménagées en bois et en terre, ou en tout-venant. Jamais en maçonnerie de mortier de chaux. On comprend d'ailleurs mal pourquoi les ingénieurs militaires auraient tenu à employer cette méthode à Azaila. Elle n'apporte aucune solidité ni stabilité supplémentaire à un ouvrage d'utilisation ponctuelle. En effet, la pérennité que le mortier confère à la construction n'a pas de sens dans le contexte d'un conflit armé. Elle s'accorde mal avec la fugacité du combat, même s'il s'agit d'un siège relativement long. Enfin, en termes de mise en œuvre, de coût et de rapidité d'exécution, son emploi se serait révélé plus contraignant que celui de la terre. Rappelons, en effet, que pour réaliser le mortier, il aurait d'abord fallu construire les fours dans lesquels on aurait réduit le calcaire, le gypse et l'albâtre en chaux. Même si la nature friable de la roche locale rendait cette opération moins longue qu'à l'accoutumée, il aurait néanmoins fallu attendre

88 Le volume de matériaux employé est estimé à environ 8 ou $10000 \mathrm{~m}^{3}$ (Romeo, 2004: 41).

89 Cabré, 1944. Il dit aussi avoir mis au jour quelques rares poches de charbons de bois et d'argile, sans doute des adobes.

90 Beltrán Lloris, 1976; Beltrán Lloris et al., 1995: 175; Saéz Abad, 2005: 161.

91 Il n'y a aucune raison de douter sérieusement de la nature de cette maçonnerie. De toute évidence, on peut compter sur l'expérience des ouvriers de fouille du début du XX $\mathrm{XX}^{\mathrm{e}}$ siècle pour savoir reconnaître une maçonnerie de mortier. Néanmoins, par précaution, on doit rappeler que les matériaux d'origine locale employés dans les remblais —il s'agit essentiellement de blocs de gypse et d'albâtre — ont la particularité de se dissoudre facilement au cours des siècles. Ils forment alors, de façon naturelle, un liant compact qui ressemble au mortier de chaux.

92 Adam, 1984: 82-83.

93 C'est le cas des parties hautes de la muraille nord-est d'Olèrdola (San Miguel, Olèrdola, Barcelone) (Batista et al., 1989-1990) et de celle de la «ville romaine» d'Ampurias (Turó d'Empúries, Ampurias, Gérone) (Mar et Ruiz, 1993), par exemple.

${ }_{94}$ De plus, dans la majorité des cas, l'opus caementicium d'époque républicaine est d'une texture différente de celui employé dans le talus d'Azaila. Les pierres sont peu nombreuses et le mortier très sableux, voire terreux.

95 Ceci s'explique essentiellement par le type d'outillage auquel les soldats avaient accès. Les pierres n'étaient d'ailleurs que très rarement travaillées. Cette opération nécessite en effet un savoir-faire et des outils particuliers.

96 Voir, par exemple, Vitruve X, 13, 3, 6 pour les rampes en bois mobiles qui permettaient le passage de plainpied sur un rempart et $X, 16,12$ pour les rampes d'assaut supportant les tours mobiles. Pour ces dernières, voire aussi César, $B C, 2,1$ et 2, 15 et $B G, 7,22$ et 7,24. Généralement, la structure extérieure de la rampe, nécessaire au maintien des remblais sous la pression des lourdes machines d'artillerie, était construite en bois.

97 Voir Campbell 2003a, 2003b et 2005. L'exemple le plus célèbre de rampe d'assaut construite lors du siège d'une ville dans le monde antique est bien évidemment celui qui conduisit à la prise de Massada au printemps 74 p.C. (Avi-Yonah et al., 1957; Yadin, 1967; Cordente Vaquero, 1992). L'épisode est conté par Flavius Josèphe (BI, VII, 28). 


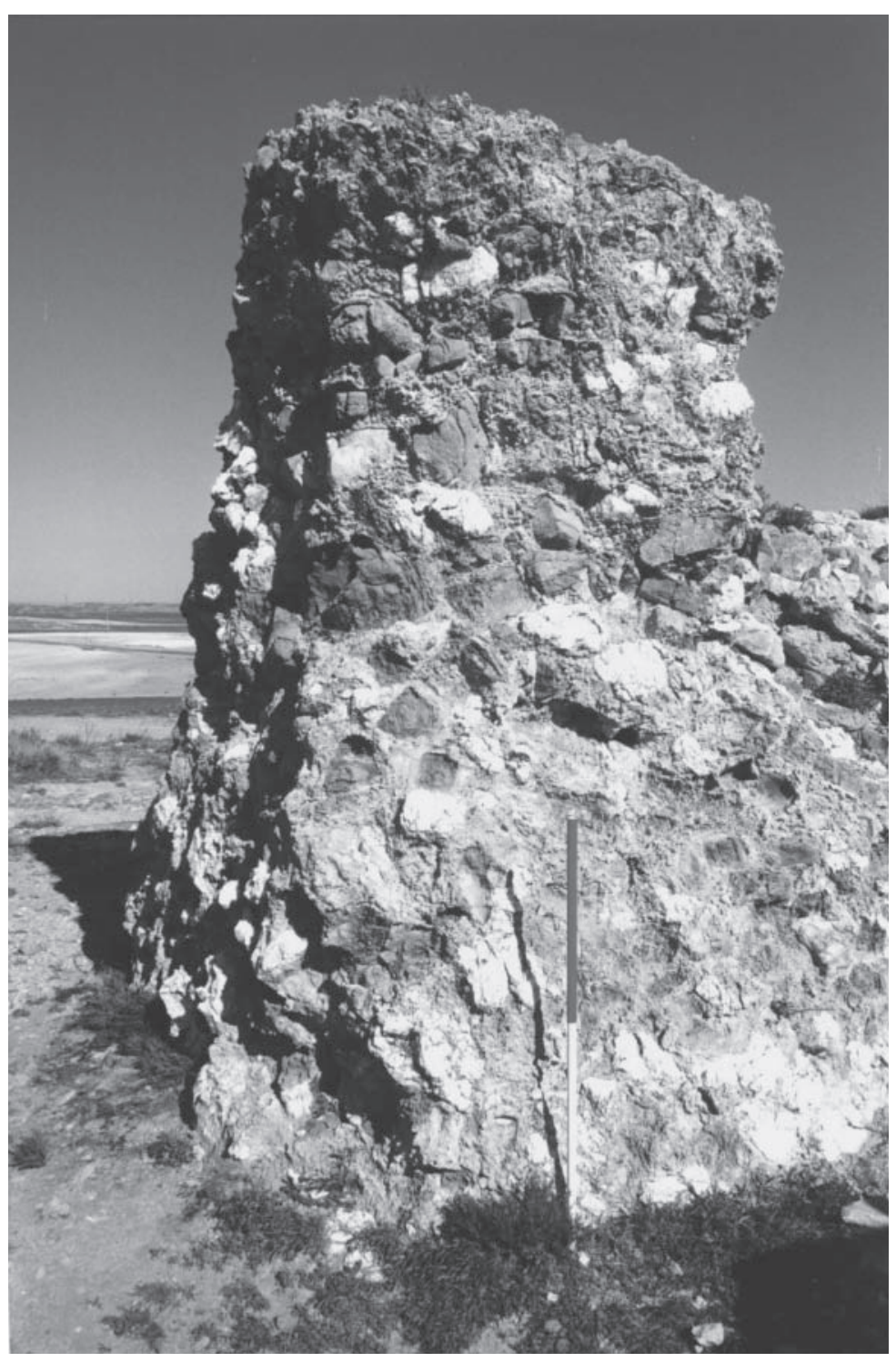

Figure 10. Vue du remplissage de la «rampe» d'Azaila (cl. D. Hourcade).

au moins deux jours supplémentaires pour la créer ${ }^{98}$. Si on ajoute à cela le temps de préparation du mortier, puis le temps de séchage de la maçonnerie, on comprend que, pour l'aménagement de cette rampe, il aurait été plus rapide, plus simple et tout aussi efficace de n'utiliser que la terre et les pierres disponibles à proximité.

De plus, dans le remplissage de ce talus, deux autres éléments me paraissent également étonnants: 1'existence, en partie basse, d'une couche de remblai visiblement différente de la

98 Sur le processus de transformation du calcaire en chaux et la préparation du mortier, voir Adam, 1984: 69-82. La création du plâtre est plus rapide. L'utilisation du gypse permet en effet de réduire considérablement les temps de cuisson (Adam, 1984: 73). 
maçonnerie d'opus caementicium, d'une part, et la faible présence de débris de démolition, de l'autre.

Les photographies prises à l'époque des fouilles de Cabré montrent toutes, en effet, que la partie inférieure du talus est composée d'une couche de remblai relativement épaisse et homogène (fig. 9). Elle recouvre en fait le sommet des «pierres plantées» et semble créer un niveau relativement plat, ou du moins peu incliné ${ }^{99}$. On remarque d'ailleurs que c'est apparemment cette même couche de remblai qui comblait la partie du fossé située sous le talus ${ }^{100}$. Cette différence stratigraphique implique, selon moi, l'existence de deux phases chronologiques distinctes ${ }^{101}$. Il ne peut s'agir de deux étapes d'un même chantier. En effet, on a du mal à concevoir que les soldats romains aient d'abord commencé à aménager le talus à l'aide des matériaux disponibles à proximité, puis qu'ils se soient compliqué la tâche en leur superposant une maçonnerie d'opus caementicium. Il aurait été plus simple et plus stable d'élever la totalité de la rampe avec les mêmes matériaux ${ }^{102}$. En fait, je serais tenté de croire que la couche inférieure du talus correspond aux niveaux d'abandon du site et, plus particulièrement, de ce quartier de la ville basse. Je pense que c'est l'érosion naturelle, ainsi que le ruissellement des couches provenant du sommet et des versants de la colline qui ont concouru à créer ce premier niveau de remblai qui recouvre et nivelle les vestiges antiques. La maçonnerie de mortier de chaux ne serait ajoutée que dans un second temps.

Un autre argument me semble également plaider en faveur d'une structure postérieure à un hypothétique siège de la ville. C'est l'absence, ou du moins la relative rareté, de mobilier archéologique et de témoins des niveaux de démolition. En effet, la maçonnerie d'opus caementicium semble entièrement composée de matériaux de nature ou d'origine géologique (fig. 10). Or, si cette rampe avait été construite lors d'un conflit, elle aurait aussi été aménagée avec les débris des habitats et des entrepôts sur lesquels elle devait s'élever ${ }^{103}$. Au pire, elle les aurait fossilisés. Ce n'est pas le cas ici. Ils sont quasiment absents des niveaux inférieurs ${ }^{104}$. Au final, tout se passe comme si ce talus avait été élevé avec des matériaux préalablement triés, ou provenant d'une autre zone, à une époque où les débris des constructions de la ville basse n'étaient plus accessibles. Rien dans ces caractéristiques ne rappelle donc celles d'une rampe d'assaut construite au cours d'un siège.

La forme de ce talus prête aussi à discussion (fig. 11). Il ne ressemble en effet que peu à ce que l'on sait des autres rampes d'assaut antiques. Long d'au moins $75 \mathrm{~m}$ et large d'environ 26 ou 27 m, il n'atteint que 4,20 m de hauteur au maximum. C'est peu. Sa forme primitive

99 Cette stratigraphie demeure hypothétique et aucune étude ne mentionne expressément l'existence de cette couche inférieure. Néanmoins, le fait que les ouvriers de Cabré aient réussi à dégager sans grande difficulté les contours des blocs fichés en terre prouve, à mon sens, que la couche qui les recouvrait est essentiellement composée de terre et de pierres. S'il s'était agi d'une maçonnerie de mortier de chaux, le dégagement des blocs aurait été en grande partie impossible.

${ }^{100}$ Ni les notes de J. Cabré, ni le relevé effectué par M. Beltrán Lloris (Beltrán Lloris et al., 1995, fig. 91), ne permettent en effet de penser que le remblai d'opus caementicium recouvrait le fond du fossé (fig. 11). Il faut donc en déduire que, comme au niveau des «pierres plantées», cette maçonnerie a été installée sur le sommet d'une couche de remblai différente et antérieure.

${ }^{101}$ Cette remarque donne également moins de poids à l'hypothèse formulée par Cabré au sujet des blocs plantés. Selon lui, ils auraient servi à stabiliser les remblais du «tumulus». Il me semble pourtant que, si tel était le cas, ils auraient alors été noyés dans la masse de mortier et non recouverts par une première couche de tout-venant.

102 On imagine mal, également, que les habitants de la ville soient responsables de ce premier nivellement. Ils n'auraient fait que faciliter l'accès des ennemis à l'acropole.

103 Voir les remarques de Garlan, 1974: 142-143.

${ }^{104}$ En fait, les fouilles de Cabré ont permis la mise au jour de certains restes, mobiliers et immobiliers, de ces habitats (Cabré, 1944). Mais, puisque la couche supérieure du talus semble clairement stérile, il est fort probable qu'ils proviennent de la couche inférieure. Ils sont d'ailleurs bien peu nombreux au regard de ce que l'on serait en droit d'attendre si cette rampe avait recouvert un quartier encore habité quelques jours avant sa construction. 
demeure en grande partie inconnue. Cabré considérait qu'il avait la forme d'un dôme. C'est en partie ce qui l'avait conduit à y voir les vestiges d'un tumulus funéraire. Cependant, le profil réalisé par Beltrán Lloris laisse plutôt penser qu'il s'agissait à l'origine d'un plan incliné (fig. $11)^{105}$. Son extrémité nord — de part et d'autre du fossé et au contact avec la muraille sudaurait disparu sous l'effet de l'érosion, ou au cours des fouilles anciennes. D'après les relevés, le pendage originel du talus aurait été d'environ 4 à $6 \%$, c'est-à-dire environ 6 degrés. Cet an-

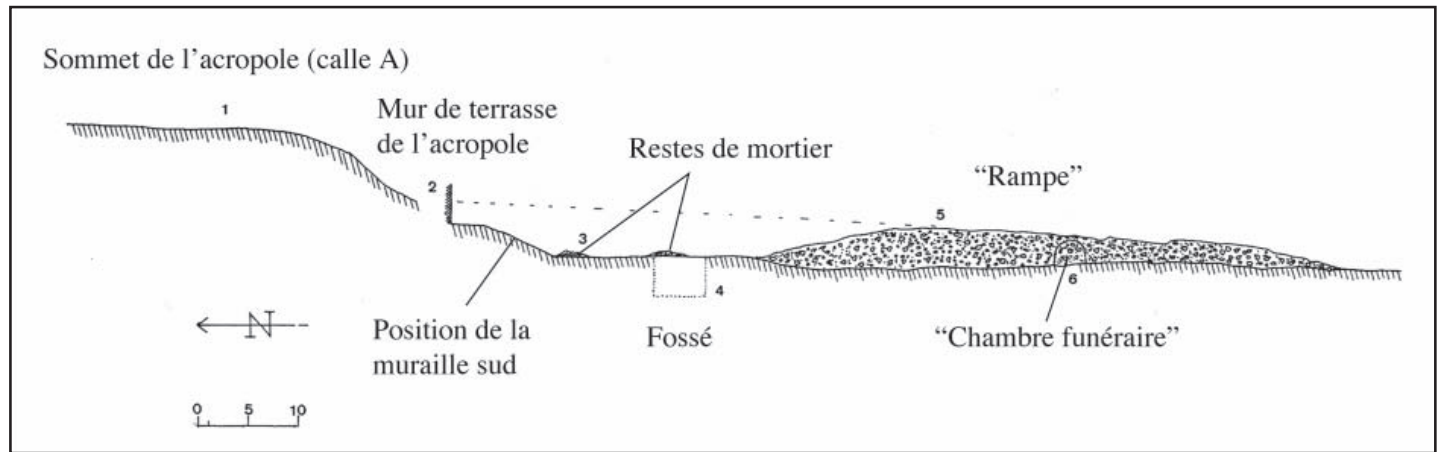

Figure 11. Profil longitudinal de la «rampe» d'Azaila (d’après Beltrán Lloris et al., 1995: fig. 91).

gle me parait étrangement faible pour une rampe d'assaut. Il crée en fait une pente très douce qui n'a rien de comparable avec l'inclinaison des rampes de Massada, Marseille ou Avaricum (Bourges), par exemple ${ }^{106}$. Dans tous ces cas, le pendage est cinq à six fois supérieur à celui du talus d'Azaila.

De plus, il me semble que l'on doit s'interroger sur l'intérêt qu'auraient eu les assaillants à construire un tel ouvrage. Était-il vraiment nécessaire au regard des défenses de la ville et de la topographie du site ? En effet, les rampes d'assaut sont normalement construites pour permettre aux machines de siège de s'avancer au plus près des remparts, d'attaquer le sommet des murailles — c'est-à-dire les parties les plus fragiles - et de surplomber les assiégés ${ }^{107}$. Elles servent aussi à compenser les désavantages d'un relief trop prononcé. Or, à Azaila, rien dans les défenses de la ville, ni dans la topographie, ne rendait nécessaire un tel ouvrage. On l'a

${ }^{105}$ Beltrán Lloris et al., 1995, fig. 91 = fig. 11. Le fait que l’on ait repéré des traces de mortier ( $\mathrm{n}^{\circ}$. 3) jusqu'au pied de la muraille sud permet de penser que le talus s'étendait au moins jusque-là. À moins que ces dépôts soient d'origine secondaire et qu'ils soient dus à l'érosion, on doit donc en conclure que les deux tiers des terres de ce remblai se situaient au nord de la probable chambre funéraire mise au jour par Cabré ( $\mathrm{n}^{\circ}$. 6). Dans ces conditions, il me semble peu probable que l'on puisse encore voir dans ce talus les restes d'un tumulus. Si cela avait été le cas, son dôme aurait été centré au-dessus de la chambre funéraire et non pas décalé vers le nord, comme c'est le cas ici (fig. 11). Une mise en garde s’impose néanmoins. Sur le plan de Cabré, la chambre funéraire est dessinée au centre de l'espace compris entre le mur d'escarpe du fossé et l'extrémité sud du talus (fig. 1). Dans l'optique d'un tumulus, sa position serait donc parfaite. S'agit-il d'une erreur de relevé ? Seules de nouvelles mesures permettraient de résoudre cette question.

${ }^{106}$ Même si la topographie du site de Massada n'est en rien similaire avec celle de l'agglomération ibère, on ne peut être que frappé par les différences qui existent entre les dimensions des deux talus. À Massada, la rampe d'assaut mesure $225 \mathrm{~m}$ de long et près de $75 \mathrm{~m}$ de hauteur. Son pendage est donc extrêmement prononcé. Il est de 33,3\% ! (Avi-Yonah et al., 1957: 39-45; Cordente Vaquero, 1992: 165-167). À Marseille, la rampe d'assaut construite par César en 49 a.C. atteignait $24 \mathrm{~m}$ de hauteur (BC, 2, 1 et 2, 15). Celle construite devant Avaricum mesurait 330 pieds de long et 80 pieds de haut. Son pendage était donc de $24 \%(B G, 7,22$ et 7,24$)$.

107 Garlan, 1974: 142-143. 
vu plus haut, les «pierres plantées» pouvaient être contournées ou rapidement nivelées, alors que le fossé aurait aisément pu être en partie comblé. Enfin, il est probable que la hauteur de la muraille sud, qui fait face à la rampe, ne devait pas excéder $8 \mathrm{~m}$. Or, on sait que les échelles et les tours d'assaut mobiles construites par les troupes romaines dépassent généralement, à elles seules, ces dimensions ${ }^{108}$. De plus, si les relevés et les calculs effectués par Beltrán Lloris sont exacts, on est forcé d'admettre que le gain de hauteur permis par cette rampe aurait été ridiculement faible au regard des travaux nécessaires. Si le pendage du talus était constant, tout indique que, au pied de la muraille sud, comme à celui du mur de terrasse de l'acropole, la rampe ne devait plus mesurer que $3 \mathrm{~m}$ de hauteur au maximum (fig. 11). C'est évidemment trop peu pour une rampe d'assaut ${ }^{109}$. Au final, ce chiffre permet donc, à mon sens, de récuser définitivement l'hypothèse formulée au sujet de ce talus. Il ne peut pas s'agir d'un ouvrage militaire.

À quoi pouvait-il donc servir? La question reste ouverte. Cependant, je pense qu'il pourrait en fait s'agir d'une rampe d'accès construite dans un but pratique et pacifique. On l'a dit, en effet, son inclinaison est faible. Or, elle est en réalité parfaite pour permettre à des charrettes, même lourdement chargées, d'accéder facilement au sommet de la colline. De plus, il est frappant de voir que le gain de hauteur réalisé par ce talus permet de communiquer de plain-pied avec la rue antique qui mène à l'acropole (fig. 11) ${ }^{110}$. En fait, tout se passe comme si ce talus ne faisait que prolonger vers le sud, avec les mêmes pendage et orientation, l'ancienne rue principale de l'agglomération (fig. 1). On comprend alors mieux la position de cette rampe. Elle ne cherche pas à mettre à profit une fragilité défensive, mais, au contraire, à tirer parti du relief et de l'urbanisme préexistant. Par cette rampe, on crée une rue presque rectiligne et en pente douce. Les véhicules évitent ainsi le virage très prononcé de l'ancienne rue qui passait par la porte méridionale (fig. 5).

Reste alors le problème de la datation de cette rampe. Faute de données stratigraphiques précises et de mobilier mis au jour dans les terres du remblai, on ne peut proposer aucune datation absolue ${ }^{111}$. On sait cependant qu'il ne peut être que contemporain ou postérieur à l'abandon du site. Il recouvre en effet les rues et les habitats d'un quartier de la ville basse et en condamne l'usage ${ }^{112}$. Si, pour les raisons que j'ai exposées plus haut, on récuse l'hypothèse d'une rampe d'assaut construite lors d'un siège et qu'on lui préfère celle d'une rampe d'accès aménagée pour permettre aux charrettes d'atteindre aisément le sommet de la colline, il me semble que l'on peut préférer deux périodes pour la datation de cette structure. La première est celle de la fin du Ir siècle a.C., alors que la seconde, plus large, correspond à un moment indéterminé du Moyen Âge ou de l'époque moderne. C'est en effet à ces deux époques que l'agglomération antique a vraisemblablement servi de carrières d'extraction de matériaux. Si

\footnotetext{
${ }^{108}$ C'est ce que rapporte Vitruve, X, 13, 3-5. Voir aussi Marsden, 1971; Campbell, 2005.

109 Tous les exemples cités plus haut montrent que les rampes atteignent normalement plusieurs dizaines de mètres de hauteur.

${ }^{110}$ Le profil réalisé par Beltrán Lloris (fig. 11) est en ce sens extrêmement clair. On y voit parfaitement que le sommet de la rampe se situe à la même cote que la partie intermédiaire de la rue principale. Il ne faut pas, d'ailleurs, se laisser abuser par la restitution. Contrairement à ce que pourrait faire penser le relevé, le mur $\mathrm{n}^{\circ} .2$ ne correspond pas au rempart de la ville. Il s'agit du mur de terrasse de l'acropole. La muraille sud, qui se situe plus en contrebas, est, à cet endroit, aujourd'hui entièrement arasée. Rien ne dit qu'elle jouait encore un rôle d'obstacle au moment où la rampe a été construite. Cela me paraît très peu probable.

${ }^{111}$ Les arguments avancés plus haut demeurent des hypothèses de travail. Il faudrait, là aussi, envisager l'ouverture de nouveaux sondages archéologiques pour résoudre définitivement ce problème.

112 En fait, comme je l'ai expliqué auparavant, il est probable que cette condamnation soit antérieure à la maçonnerie d'opus caementicium. Selon moi, elle date de la création de la couche inférieure repérée dans le talus. Le $T P Q$ est donné par le mobilier provenant des derniers niveaux d'occupation, c'est-à-dire les environs des années 40 a.C. (Gorgues, 2005; Ribera et Marín, 2004-2005).
} 
cette interprétation reste hypothétique dans le premier cas ${ }^{113}$, elle est avérée pour le second. On sait en effet que les pierres du Cabezo de Alcalá ont servi à la construction du bourg moderne d'Azaila ${ }^{114}$. Peut-être est-ce à cette époque seulement que cette rampe a été construite. Si c'est le cas, elle témoignerait donc non pas d'un siège, mais de la volonté d'accéder au site pour y récupérer des matériaux de construction, plusieurs siècles après l'abandon de la ville. Il n'est d'ailleurs pas exclu que cette rampe et le chemin qu'elle dessine aient été utilisés jusqu'à la fin $\mathrm{du} \mathrm{XIX}^{\mathrm{e}}$ siècle, voire au début du $\mathrm{XX}^{\mathrm{e}}$ siècle. Le relevé de Cabré est, à ce sujet, étrangement explicite. En effet, l'extrémité nord de la rampe et l'ancienne rue qui conduit à l'acropole sont reliées par une flèche à côté de laquelle figure la mention «primitiva entrada» (fig. 1).

En conclusion de cette étude méthodologique, je pense que, malgré l'apparente accumulation d'《évidences» archéologiques, on doit désormais douter de la réalité du siège d'Azaila. Selon toute vraisemblance, il n'a jamais eu lieu. Ou plutôt, si cela fut le cas, il ne s'est déroulé ni à la date généralement proposée, ni dans les circonstances évoquées. Il n'a pas été fatal à la ville. Plus important encore, on peut préférer une lecture pacifique, démilitarisée, pratique et civique de l'ensemble des arguments utilisés pour supposer l'existence du siège.

Ainsi, même s'il est probable que les blocs fichés en terre découverts en avant du fossé soient des lignes de défense de type «pierres plantées», rien ne dit qu'elles témoignent d'une guerre. Elles pourraient plutôt dater de la phase d'aménagement des premières fortifications de la ville. De même, les «barricades» repérées en travers des rues de l'acropole ne sont peut-être que des pierriers d'époque moderne. Ils pourraient dater du temps où le site a été transformé en carrière. De plus, on sait que les deux catapultes de torsion et les armes de poing — censées prouver l'âpreté du combat et la résistance des habitants - ne sont que d'anciens trophées exposés et abandonnés. Ils témoignent certainement d'un conflit, mais sa date et sa localisation restent indéterminées. Parallèlement, rien ne prouve que les flèches et les projectiles de pierre découverts sur l'acropole aient servi. Ils pourraient provenir de l'arsenal municipal. Enfin, il est probable que le talus situé au sud de la ville haute ne soit pas une rampe d'assaut construite par les troupes romaines au moment de prendre la ville, mais une simple rampe d'accès. Elle pourrait avoir été aménagée postérieurement à l'abandon du site pour rendre l'accès au sommet de la colline plus aisé. Au final, les armes et les défenses mises au jour ne me semblent pas témoigner de la mort du site. Au contraire, elles nous rappellent les différentes étapes de sa vie, depuis sa fondation jusqu'à son abandon à la fin de la République, voire sa transformation en carrières à l'époque moderne.

Comment, dans ces conditions, expliquer l'abandon du site aux alentours des années 40 a.C. ? La raison est certainement avant tout politique. Elle implique en effet l'ensemble de la communauté. Rien ne prouve cependant qu'il se soit agit d'une déportation forcée, ni de la conséquence d'un drame militaire ${ }^{115}$. En fait, le départ a pu être décidé par les autorités locales ou, du moins, avec leur accord. Il est également possible de trouver une autre raison d'ordre pratique. Peut-être que le site d'Azaila n'était alors plus en adéquation avec les réalités économiques et géopolitiques de l'époque. Nul doute d'ailleurs que la fondation, à la même époque,

113 On peut en effet imaginer que, lors de la fondation de la nouvelle colonie Victrix Iulia Lepida (Celsa) sur le site voisin de «Las Eras» (Velilla del Ebro, Saragosse), les architectes antiques aient préféré remployer certains matériaux provenant d'Azaila. Ils auraient ainsi pu réduire le temps et les coûts de construction.

114 Cabré, 1944; Beltrán Lloris et al., 1995.

115 Sur ce sujet, on se souviendra des mises en garde des chercheurs médiévistes. P. Toubert rappelle ainsi que «même la chronicité de la guerre n'explique pas les abandons (...). Les désertions d'habitats du monde méditerranéen sont très rarement des phénomènes foudroyants. Un habitat meurt rarement d'une crise cardiaque, mais plutôt d'une maladie plus chronique» (Toubert, 1988: 9). 
de la nouvelle colonie Victrix Iulia Lepida («Las Eras», Velilla del Ebro, Saragosse) — située à une dizaine de kilomètres au nord-ouest et en bordure de l'Ėbre - ait joué un rôle dans l'abandon du site d'Azaila. Elle en est soit la cause, soit le résultat.

Au final, même si plusieurs des interprétations formulées dans cet article ne sont que des hypothèses - dont certaines demeureront à jamais invérifiables-, elles permettent néanmoins de proposer une alternative plus conforme à ce que l'on sait de l'abandon des autres sites de la région et de la péninsule. Elles rappellent aussi que, malgré une conquête longue et parfois difficile, la romanisation de la péninsule Ibérique ne s'est pas uniquement faite dans le sang.

\section{BIBLIOGRAPHIE}

ADAM, J. P. (1984): La construction romaine antique: matériaux et techniques. Paris.

Aдсоск, F. E. (1940): The Roman Art of War under the Republic. Cambridge.

Alonso, N. et al. (éds.) (2003): Chevaux-de-frise i fortificació en la primera edat del ferro europea. Lérida.

Asensio Esteban, J. Á. (1995): La ciudad en el mundo prerromano en Aragón. Zaragoza.

Avi-Yonah, M., Avigad, N., Aharoni, Y, Dunayevsky, I. et Guttman, S. (1957): Masada. Survey and excavations, 1955-1956. Jerusalem.

Barrandon, N. (2005): De la conquête à la pacification. Les mutations des sociétés indigènes et les débuts de la romanisation en Hispanie septentrionale et centrale de la chute de Numance aux guerres asturo-cantabres (133-26 avant J.-C.). Thèse inédite, Université de Bordeaux 3, Bordeaux.

Batista, R., Molist, N. et Rovira, J. (1989-1990): «El conjunt monumental d'Olèrdola: les darreres campanyes d'excavacions (1983-1989)». Tribuna d'Arqueologia: 87-99.

Beltrán Lloris, M. (1976): Arqueología e historia de las ciudades antiguas del Cabezo de Alcalá de Azaila (Teruel). Zaragoza.

Beltrán Lloris, M. (1984a): «Don Juan Cabré y Azaila: estado actual de conocimiento del Cabezo de Alcalá (Teruel)», Juan Cabré Aguiló (1882-1982): encuentro de homenaje. Zaragoza: 79-92.

Beltrán Lloris, M. (1984b): «Nuevas aportaciones a la cronología de Azaila». Boletín del Museo de Zaragoza, III: 125-152.

Beltrán Lloris, M. (1990): «Roma: República y Alto Imperio», Estado actual de la arqueología en Aragón. Vol. I: Ponencias. Zaragoza: 215-262.

Beltrán Lloris, M. (éd.) (1987): Arcóbriga (Monreal de Ariza, Zaragoza). Zaragoza.

Beltrán Lloris, M., Mostalac, A. et Guiral, C. (1995): Azaila. Nuevas aportaciones deducidas de la documentación inédita de Juan Cabré Aguiló. Zaragoza.

Beltrán Lloris, F., Martín-Bueno, M. et Pina Polo, F. (dirs.) (2000): Roma en la Cuenca Media del Ebro. La Romanización en Aragón. Zaragoza.

Beltrán Lloris, A. (1964): «Notas sobre la cronología del poblado del Cabezo de Alcalá, en Azaila (Teruel)». Caesaraugusta, XXIII-XXIV: 79-86.

Beltrán Martínez, A. (1966): «Sobre la cronología de Azaila (Teruel)», IX Congreso Nacional de Arqueología (Valladolid, 1965). Zaragoza: 308-309.

Beltrán Martínez, A. (1987): «Los asentamientos ibéricos ante la romanización en el Valle del Ebro: los casos de Celsa, Azaila y Botorrita», Los asentamientos ibéricos ante la romanización. Madrid: 101-110.

Benavente, J. A., Gorgues, A., Marco Simón, F. et Moret, P. (2004): «Les campagnes de fouilles 2003 et 2004 à El Palau (Alcañiz, Teruel)». MCV, XXXIV-II: 358-370.

Berrocal-Rangel, L. et Moret, P. (2007): «Las fortificaciones protohistóricas de la Hispania céltica: cuestiones a debate», L. Berrocal-Rangel et P. Moret (éds.), Paisajes fortificados de la Edad del Hierro. Madrid: 15-33.

Bosh Gimpera, P. (1913-1914): «La catapulta d'Empuries». Anuari de l'Institut d'Estudis Catalans, II: 105-111.

Burillo, F. (1986): Aproximación diacrónica a las ciudades antiguas del valle Medio del Ebro. Teruel. 
CABré, J. (1925): «Los bronces de Azaila». AEspA, I: 297-315.

CABré, J. (1929): «Azaila», IV Congrès International d'Archéologie. Barcelone: 6-14.

CABré, J. (1941): «La acrópolis de Alcalá, Azaila (Teruel)». AEspA, XLII: 232-235

CABré, J. (1944): Corpus Vasorum Hispanorum. La cerámica de Azaila. Madrid.

Campbell, D. B. (2003a): Greek and Roman Siege Machinery 399 BC-AD 363. Osprey New Vanguard, LXXVIII. Oxford.

Campbell, D. B. (2003b): Greek and Roman Artillery 399 BC-AD 363. Osprey New Vanguard, LXXXIX Oxford.

CampBell, D. B. (2005): Siege Warfare in the Roman World, 146 BC-AD 378. Oxford.

Cinca, J. L., Ramírez Sádaba, J. L. et Velaza, J. (2003): «Un depósito de proyectiles de catapulta hallado en Calahorra (La Rioja)». AEspA, LXXVI: 263-271.

Cordente Vaquero, F. (1992): «La toma de Masada: ejemplo de eficacia de la técnica poliorcética en el éjercito romano». Gerion, X: 155-170.

DíAz AriÑo, B. (2005): «Glandes inscriptae de la Península Ibérica». Zeitschrift für Papyrologie und Epigraphik, CLIII: 219-236

Franco Calvo, J. G. et Hernández Pardos, A. (2004-2005): «El Cabezo de Alcalá de Azaila: Avance de resultados de los trabajos arqueológicos realizados el año 2003». Kalathos, XXII-XXIII: 369-377.

Gabaldón Martínez, M. (2004): Ritos de Armas en la Edad del Hierro. Armamento y lugares de Culto en el Antiguo Mediterráneo y el mundo celta. Anejos de Gladius, VII, Madrid.

Gailledrat, E. et Moret, P. (2003): «La fortification de Pech Maho (Sigean, Aude) et le problème de ses pierres plantées», N. Alonso, E. M. Junyent, A. Lafuente et J. B. López (éds.), Chevaux-de-frise i fortificació en la primera edat del ferro europea. Lérida: 119-133.

García Díez, F. (2002): «Las catapultas de Azaila (Teruel)», A. Morillo (dir.), Arqueología militar romana en Hispania. Anejos de Gladius, V, Madrid: 293-302.

Garlan, Y. (1974): Recherches de poliorcétique grecque. Paris.

Gorgues, A. (2005): Économie et société dans le nord-est du monde ibérique et ses marges (250/25 av. $J$.-C.). Thèse inédite, Université de Toulouse-Le Mirail, Toulouse.

Gracia Alonso, F. (1997): «L'artillerie romaine et les fortifications ibériques dans la conquête du NordEst de la péninsule ibérique», M. Feugère (éd.), L'équipement militaire et l'armement de la république (IV e $^{e r}$ s. av. J.-C.). JRMES, VIII: 201-231.

Gracia Alonso, F. (2000): «Análisis táctico de las fortificaciones ibéricas». Gladius, XX: 131-170.

Gracia Alonso, F. (2001): «Sobre fortificaciones ibéricas. El problema de la divergencia respecto al pensamiento único». Gladius, XXI: 155-166.

Gracia Alonso, F. (2003): La guerra en la Protohistoria. Héroes, nobles, mercenarios y campesinos. Barcelona.

Gracia Alonso, F. (2006): «Las fortificaciones ibéricas: análisis poliorcético y concepto de empleo táctico en la guerra de sitio», A. Oliver Foix (dir.), Arquitectura defensiva: la protección de la población y del territorio en época ibérica. Castellón: 63-122.

Harbison, P. (1971): «Wooden and stone chevaux-de-frise in central and western Europe». Proceedings of the Prehistoric Society, XXXVII: 195-225.

HourCADE, D. (2003): «Les murailles des villes romaines de l'Hispanie républicaine et augustéenne: enceintes ou fortifications du territoire urbain?», A. Morillo, F. Cadiou et D. Hourcade (éds.), Defensa y territorio en Hispania de los Escipiones a Augusto. Madrid-León: 297-324.

HourCADE, D. (à paraître): «Les “évidences” archéologiques de siège et de prise de villes dans l'Hispanie républicaine: quelques faux indices», F. Cadiou, M. Navarro et A. Magallón (éds.), La guerre et ses traces dans la péninsule Ibérique à l'époque de la conquête romaine (Actes de la Table Ronde, Casa de Velázquez, Madrid, 23-24 novembre 2007). Zaragoza-Bordeaux.

Mar, R. et Ruiz de Arbulo, J. (1993): Ampurias romana. Historia, Arquitectura y Arqueología. Sabadell.

Marsden, E. W. (1969): Greek and Roman Artillery. Historical Development. Londres.

Marsden, E. W. (1971): Greek and Roman Artillery. Technical treatises. Londres.

Moret, P. (1996): Les fortifications ibériques de la fin de l'Âge du Bronze à la conquête romaine. Madrid.

Moret, P. (2001): «Del buen uso de las murallas ibéricas». Gladius, XXI: 137-144.

Nony, Cl.-J. (1969): «Une nouvelle interprétation des bronzes d'Azaila». MCV, V: 5-26. 
Pesez, J.-M. et Piponnier, F. (1988): «Traces matérielles de la guerre sur un site archéologique», A. Bazzana (éd.), Castrum 3. Guerre, fortification et habitat dans le monde méditerranéen au Moyen Âge. Madrid-Rome: 11-16.

Pina Polo, F. et Zanier, W. (2006): «Glandes inscriptae procedentes de la Hispania Ulterior». AEspA, LXXIX: 29-50.

Quesada SAnZ, F. (2001): «En torno al análisis táctico de las fortificaciones ibéricas. Algunos puntos de vista alternativos». Gladius, XXI: 145-154.

Quesada Sanz, F. (2003): «De los fosos de Troya a la línea Sigfrido. Las "piedras hincadas” en el contexto de la historia de las fortificaciones», N. Alonso et al. (éds.), Chevaux-de-frise i fortificació en la primera edat del ferro europea. Lérida: 69-100.

Quesada SAnZ, F. (2007): «Asedio, sitio, asalto... Aspectos prácticos de la poliorcética en la Iberia prerromana», L. Berrocal-Rangel et P. Moret (éds.), Paisajes fortificados de la Edad del hierro. Las murallas protohistóricas de la Meseta y la vertiente atlántica en su contexto europeo. Madrid: 75-98.

Ribera i Lacomba, A. et Calvo Galvez, M. (1995): «La primera evidencia arqueológica de la destrucción de Valentia por Pompeyo». Journal of Roman Archaeology, VIII: 19-40.

Ribera i LACOMBA, A. et Marín, C. (2004-2005): «Las cerámicas del nivel de destrucción de Valentia (75 a.C.) y el final de Azaila». Kalathos, XXII-XXIII: 271-300.

Richmond, I. A. (1945): Roman artillery. Oxford.

Romeo Marugán, F. (2004): «Reflexiones sobre el asedio de la ciudad ibérica del Cabezo de Alcalá de Azaila». Boletín del Museo de Zaragoza, XVIII: 25-54.

Rovira Hortala, M. C. (1999): «Las armas-trofeo en la cultura ibérica: pautas de identificación e interpretación». Gladius, XIX: 13-32.

Ruiz Zapatero, G. (2003): «Las fortificaciones de la Primera Edad del Hierro en la Europa templada», N. Alonso et al. (éds.), Chevaux-de-frise i fortificació en la primera edat del ferro europea. Lérida: 13-34.

SÁEz Abad, R. (2003): «La Poliorcética. El éxito asegurado en las operaciones de asedio». Espacio, Tiempo y Forma, Serie II, XVI: 19-40.

SÁez Abad, R. (2005): Artillería y poliorcética en el mundo grecorromano, Anejos de Gladius. VIII, Madrid.

SÁez AbAd, R. (2006): «Un siglo de hallazgos vinculados a la maquinaria bélica en Hispania», A. Morillo (éd.), Arqueología militar romana en Hispania. II. Producción y abastecimiento en el ámbito militar. León: 493-502.

Sauvage, M. (1991): «Le siège des villes fortifiées». Les Dossiers d'Archéologie, CLX: 56-63.

SILLIÈRES, P. (2001): «La maison aristocratique à l'époque républicaine, principalement dans la vallée de l'Èbre», M. Navarro et S. Demougin (éds.), Élites hispaniques. Bordeaux: 173-186.

TouBerT, P. (1988): «Introduction», A. Bazzana (éd.), Castrum 3. Guerre, fortification et habitat dans le monde méditerranéen au Moyen Âge. Madrid-Rome: 7-9.

TRILlmich, W. (1990): «Apuntes sobre algunos retratos de bronce de la Hispania Romana», M. Aznar, L. Gaztelu et C. Yllán (dirs.), Los Bronces Romanos en España. Madrid: 37-50.

Trillmich, W. (1997): «El modelo de la metrópoli», J. Arce, S. Ensoli et E. La Rocca (éds. lit.), Hispania Romana. Desde tierra de conquista a provincia del Imperio. Madrid: 131-141.

Vicente, J. D., Punter, M. P. et EzQuerra, B. (1997): «La catapulta tardo-republicana y otro equipamiento militar de La Caridad (Caminreal, Teruel)», M. Feugère (éd.), L'équipement militaire et l'armement de la république (IVE-Ier s. av. J.-C.). JRMES, VIII: 167-199.

YADIN, Y. (1967): Massada, la dernière citadelle d'Israël. Paris. 